\title{
Centralizer theory for long-lived spin states
}

\author{
Christian Bengs ${ }^{1, a)}$ \\ School of Chemistry, Southampton
}

(Dated: June 1, 2021)

Nuclear long-lived spin states represent spin density operator configurations that are exceptionally well protected against spin relaxation phenomena. Their long-lived character is exploited in a variety of Nuclear Magnetic Resonance (NMR) techniques. Despite growing importance of long-lived spin states in modern NMR strategies for their identification have changed little over the last decade. The standard approach heavily relies on a chain of group theoretical arguments. In this paper we present a more streamlined method for the calculation of such configurations. Instead of focusing on the symmetry properties of the relaxation superoperator, we focus on its corresponding relaxation algebra. This enables us to analyse long-lived spin states with Lie algebraic methods rather than group theoretical arguments. We show that the centralizer of the relaxation algebra forms a basis for the set of long-lived spin states. The characterisation of the centralizer on the other hand does not rely on any special symmetry arguments and its calculation is straightforward. We outline a basic algorithm and illustrate advantages by considering long-lived spin states for some spin- $1 / 2$ pairs and rapidly rotating methyl groups.

\section{INTRODUCTION}

Nuclear Magnetic Resonance (NMR) experiments investigate molecular properties through an interplay of nuclear spins and externally applied radio-frequency fields ${ }^{1,2}$. During this process the external fields inevitable perturb the nuclear spins from their thermal equilibrium position. The equilibration process may be described by a quantum master equation ${ }^{3-7}$

$$
\left.\left.\left.\frac{d}{d t} \mid \rho(t)\right)=\hat{L} \mid \rho(t)\right)=\left(\hat{L}_{\mathrm{coh}}+\hat{\Gamma}\right) \mid \rho(t)\right) .
$$

The current state of the spin ensemble is described by the density operator $\rho(t)$. The superoperator $\hat{L}$ represents the generator of motion and may be subdivided into a coherent contribution $\hat{L}_{\text {coh }}$ and a dissipative contribution $\hat{\Gamma}$. The coherent Liouvillian $\hat{L}_{\text {coh }}$ is given by the commutation superoperator of the coherent part of the Hamiltonian ${ }^{7,8}$

$$
\left.\left.\hat{L}_{\mathrm{coh}} \mid \rho(t)\right)=-i \hat{H}_{\mathrm{coh}} \mid \rho(t)\right)=-i\left[H_{\mathrm{coh}}, \rho(t)\right],
$$

The dissipative contribution $\hat{\Gamma}$ entails information about the fluctuating part of the spin Hamiltonian, but does in general not admit such a simple form. A more detailed discussion of $\hat{\Gamma}$ is therefore deferred to section II A.

The tendency of the nuclear spins to return to their thermal equilibrium position limits the duration of NMR experiments. At high magnetic fields for example, this tendency is captured by two important constants, the transverse relaxation time $T_{2}$ and the longitudinal relaxation time $T_{1}$. Several modern NMR experiments circumvent these time restrictions by utilising spin density operator configurations that are particular resilient to nuclear spin relaxation phenomena. These configurations decay

\footnotetext{
a) cb1d19@soton.ac.uk
}

with time constants which may greatly exceed typical $T_{1}$ and $T_{2}$ values $^{9-42}$.

In hindsight it is somewhat unfortunate that these density operator configurations have been referred to as "nuclear long-lived spin states", when in fact the original work by Carravetta et al. ${ }^{9}$ explored the relaxation properties of long-lived quantum mixtures rather than pure states. The term nuclear long-lived spin states should thus be interpreted in a broader sense, not referring to the relaxation properties of particular pure states, but instead to the relaxation properties of a particular state (configuration) of the physical system at hand. In order to avoid any such confusion we will instead refer to these density operator configurations as nuclear long-lived spin operators. This convention encompasses both quantum mixtures and quantum pure states.

The majority of long-lived spin operators may be found in systems with internal symmetry ${ }^{43-54}$. Typical examples include symmetric arrangements of the spin interaction network with respect spin permutations. To a first approximation the symmetric interaction network causes the relaxation process to be equally symmetric. Formally speaking the relaxation superoperator remains invariant under conjugation by some unitary superoperator $\hat{g}$

$$
\hat{g} \hat{\Gamma} \hat{g}^{\dagger}=\hat{\Gamma} \quad \text { with } \hat{g} \hat{g}^{\dagger}=\mathbb{1} .
$$

The set of superoperators $\{\hat{g}\}$ forms the symmetry group $G_{\hat{\Gamma}}$ of the relaxation superoperator.

The symmetry group of the relaxation superoperator plays a central role in the theory of long-lived spin operators. The characterisation of long-lived spin operators may then be based on group theoretical techniques ${ }^{55-57}$. Symmetry projection operators have been particularly useful in the characterisation of long-lived spin operators ${ }^{43-48,50-54}$. Nonetheless, some small caveats remain. For example, construction of symmetry projection operators can be tricky at times. Additionally, for several cases of practical relevance symmetry projection operator techniques leave large gaps between group theoretical predic- 
tions and the actual number of long-lived spin operators. Most notably for rapidly rotating methyl groups ${ }^{31,32,52}$. For such systems group theoretical arguments need to be complemented by taking rotational and permutation dual symmetries into account ${ }^{53}$.

In order to avoid some of these complications we present an alternative method for the calculation of longlived spin operators. Our approach is based on Lie algebraic methods rather than group theoretical arguments. Similar approaches have been used in the analysis of decoherence-free subspaces, which is the quantum information processing analog of long-lived spin operators $^{58-61}$. The main idea is to shift the focus from the symmetry group of the relaxation superoperator onto its relaxation algebra. A precise definition will be given below, but the relaxation algebra is closely related to the fluctuating spin interactions driving the relaxation process.

A central concept for our method will be the centralizer of the relaxation algebra. The centralizer is the set of operators that commute with all elements of the relaxation algebra simultaneously. The elements of the centralizer therefore represent spin operator configurations that are immune to relaxation. Moreover, the centralizer is closed under commutation and its elements generate a Lie subalgebra. This means that there may not exist any additional spin operators that are immune to relaxation. With this we are able to show that any long-lived spin operator may be written as a linear combination of the various elements of the centralizer. The connection between long-lived spin operators and the centralizer of the relaxation algebra offers several advantages. Most notably, a) this approach avoids any intricate symmetry arguments and $\mathbf{b}$ ) the calculation of the centralizer is straightforward. Systems displaying symmetries and dual symmetries are therefore handled in a unified manner. We outline an algorithm based on pair-wise intersections of a certain class of null spaces and illustrate the procedure by considering some simple example cases for spin- $1 / 2$ pairs and rapidly rotating methyl groups.

\section{THEORY}

\section{A. Generators of Motion}

The quantum master equation (equation 1 ) builds the basis for most of NMR relaxation theory. In order to proceed with a characterisation of long-lived spin operators a more detailed analysis of the generators $\hat{L}_{\mathrm{coh}}$ and $\hat{\Gamma}$ is necessary.

Equation 2 defines the coherent Liouvillian $\hat{L}_{\text {coh }}$ as the commutation superoperator of the coherent part of the spin Hamiltonian. The coherent spin Hamiltonian itself may be expressed as a linear combination of some spin operators $X_{m}$

$$
H_{\mathrm{coh}}=\sum_{m} x_{m} X_{m}
$$

For simplicity we assume the coefficients $x_{m}$ to be independent of time and the operators $X_{m}$ to be orthogonal with respect to the standard trace inner product

$$
\left(X_{m} \mid X_{n}\right)=\operatorname{Tr}\left\{X_{m}^{\dagger} X_{n}\right\}=\delta_{m n} .
$$

The coherent Liouvillian is therefore given by a linear combination of the corresponding commutation superoperators $\hat{X}_{m}^{7,8}$

$$
\hat{L}_{\mathrm{coh}}=-i \sum_{m} x_{m} \hat{X}_{m} .
$$

By definition, the coherent part of the Liouvillian is the same for each member of the spin ensemble. For example, in liquid state NMR the various $X_{m}$ may describe the scalar spin coupling network of the system or the interaction with a static magnetic field, whereas the coefficients $x_{m}$ describe the scalar coupling constants or the external magnetic field strength ${ }^{1,2}$.

The relaxation superoperator $\hat{\Gamma}$ is typically derived from a microscopic model. On a microscopic scale the coherent evolution of individual ensemble members is perturbed by a fluctuating spin Hamiltonian $H_{\text {fluc }}(t)$. The fluctuating Hamiltonian may be expanded as follows ${ }^{4-7}$

$$
H_{\text {fluc }}(t)=\sum_{m} y_{m}(t) Y_{m}
$$

The corresponding fluctuating Liouvillian is then simply given by

$$
\hat{L}_{\text {fluc }}(t)=-i \sum_{m} y_{m}(t) \hat{Y}_{m} .
$$

In contrast to the coherent case the expansion coefficients $y_{m}(t)$ are allowed to vary randomly in time while keeping the spin operators $Y_{m}$ fixed. In solution state NMR the various $Y_{m}$ typically describe scalar spin-spin interactions, dipolar spin interactions, etc ${ }^{4-7}$. The stochastic time dependence of the expansion coefficients $y_{m}(t)$ originates from thermal fluctuations within the liquid. For example, the distance and relative orientation of two spins may be modulated as individual molecules undergo rotational and translation Brownian motion. This in turn leads to a stochastic modulation of their dipolar coupling strength $^{62-64}$.

Although the fluctuating contributions are in general different for each ensemble member at a given point in time, the arguments summarised in appendix B show that the perturbed ensemble dynamics may approximately be described by the following evolution equation $^{4-7}$

$$
\left.\left.\frac{d}{d t} \mid \rho(t)\right)=\left\{\hat{L}_{\mathrm{coh}}-\sum_{m n} \int_{0}^{+\infty} k_{m n}(\tau) \tilde{\hat{Y}}_{m}(0) \tilde{\hat{Y}}_{n}^{\dagger}(\tau) d \tau\right\} \mid \rho(t)\right) .
$$

The randomness within the system is quantified by (stationary) correlation functions $k_{m n}(\tau)$ which describe the correlations between any two couplings parameters

$$
k_{m n}(\tau)=\overline{y_{m}(0) y_{n}^{*}(\tau)},
$$


whereas the tilde indicates a transformation into the interaction frame of the coherent Liouvillian

$$
\tilde{\hat{L}}_{\text {fluc }}(t)=\exp \left\{-\hat{L}_{\text {coh }} t\right\} \hat{L}_{\text {fluc }}(t) \exp \left\{+\hat{L}_{\text {coh }} t\right\} .
$$

A comparison with equation 1 identifies the relaxation superoperator $\hat{\Gamma}$ as a time correlated product of the fluctuating Liouvillian ${ }^{3,6,7}$

$$
\begin{aligned}
\hat{\Gamma} & =-\sum_{m n} \int_{0}^{+\infty} k_{m n}(\tau) \tilde{\hat{Y}}_{m}(0) \tilde{\hat{Y}}_{n}^{\dagger}(\tau) d \tau \\
& =\int_{0}^{+\infty} \overline{\tilde{\hat{L}}_{\text {fluc }}(0) \tilde{\hat{L}}_{\text {fluc }}(\tau)} d \tau
\end{aligned}
$$

A computationally convenient representation of $\hat{\Gamma}$ may be formulated by assuming that the operators $Y_{m}$ satisfy the relation

$$
\hat{L}_{\mathrm{coh}} Y_{m}=\left[H_{\mathrm{coh}}, Y_{m}\right]=\Omega_{m} Y_{m} .
$$

In this case, the operators $Y_{m}$ represent so-called eigenoperators of the coherent Liouvillian and the associated eigenvalues $\Omega_{m}$ may be interpreted as the transition frequencies of the system ${ }^{6,7}$. Ignoring small dynamical frequency shifts (well justified for most NMR experiments as shown in appendix C) the relaxation superoperator in equation 12 reduces to

$$
\hat{\Gamma}=-\sum_{m n} \operatorname{Re}\left\{J_{m n}\left(\Omega_{n}\right)\right\} \hat{Y}_{m} \hat{Y}_{n}^{\dagger},
$$

where we have introduced the noise spectral densities

$$
J_{m n}(\Omega)=\int_{0}^{+\infty} k_{m n}(\tau) \exp (+i \Omega \tau) d \tau
$$

The action of $\hat{\Gamma}$ onto the density operator $\rho(t)$ then reduces to a nested double commutator of the form

$$
\hat{\Gamma} \mid \rho(t))=-\sum_{m n} \operatorname{Re}\left\{J_{m n}\left(\Omega_{n}\right)\right\}\left[Y_{m},\left[Y_{n}^{\dagger}, \rho(t)\right]\right] .
$$

There are several things to note about the relaxation superoperator given by equation 14 .

1) Efficient spin relaxation may only occur for cases with spectral noise densities with sufficiently strong amplitudes at the transition frequencies of the system. This captures the fact that noise driven spin transitions are possible if the stochastic fluctuations vary on the same time scale as one of the possible transitions frequencies of the system.

2) The correlation functions $k_{m n}(\tau)$, and hence the noise spectral densities $J_{m n}(\Omega)$, are of classical nature. The relaxation superoperator $\hat{\Gamma}$ therefore represents a semiclassical approximation to the relaxation process. As a result, the spectral densities do not explicitly depend upon the temperature of the environment. Strictly speaking $\hat{\Gamma}$ describes equilibration at infinite temperature, and should be thermally corrected to account for spin relaxation at finite temperatures ${ }^{65-68}$. However, as discussed below, it is not too difficult to show that longlived spin operators at infinite temperature remain longlived within the high-temperature and high-entropy approximation. This situation applies to many conventional NMR experiments, so that we will restrict our attention to the infinite temperature case.

3) The double commutator formulation of dissipative spin dynamics may be familiar to NMR spectroscopists, but less familiar to other fields which describe open quantum systems in terms of a Lindblad equation ${ }^{69-71}$. The apparent failure of $\hat{\Gamma}$ to satisfy a Lindblad equation may be traced back to a classical treatment of the environments degrees of freedom. In particular reducing the interaction of the system and the environment to a stochastic modulation of the spin coupling parameters without any reference to the temperature of the environment. However, as discussed in reference 68 for example, it may be shown that the Lindblad equation at infinite temperature may alternatively be represented as a nested double commutator. Equation 14 should therefore be interpreted as a Lindblad equation at infinite temperature.

\section{B. Long-lived Spin Operators}

The Liouvillian $\hat{L}$ may be characterised by a set of (generalised) right eigenoperators $\mid \Phi_{j}$ ) with corresponding eigenvalues $\Lambda_{j}$

$$
\left.\left.\left.\hat{L} \mid \Phi_{j}\right)=\Lambda_{j} \mid \Phi_{j}\right)=\left(+i \omega_{j}-\lambda_{j}\right) \mid \Phi_{j}\right) .
$$

Both $\omega_{j}$ and $\lambda_{j}$ are real, with $\lambda_{j} \geq 0$ additionally being non-negative. The coherent behaviour of $\left.\mid \Phi_{j}\right)$ is characterised by its oscillation frequency $\omega_{j}$, the dissipative behaviour by its decay constant $\lambda_{j}$.

Exact long-lived spin operators, denoted by $\Phi_{j}^{0}$, have eigenvalues with a vanishing real part $\left(\lambda_{j}=0\right)$

$$
\left.\left.\left.\hat{L} \mid \Phi_{j}^{0}\right)=\Lambda_{j}^{0} \mid \Phi_{j}^{0}\right)=+i \omega_{j} \mid \Phi_{j}^{0}\right) .
$$

This definition includes long-lived populations and longlived coherences. Our definition of long-lived coherences varies from the definition given in reference 19. Sarkar et al. define long-lived coherences as coherent superpositions between different symmetry manifolds. Such coherences are in general neither eigenoperators of $\hat{L}$ nor do they display eigenvalues with a vanishing real part.

Obtaining information about the eigenoperators of a generic Liouvillian is difficult. A common starting point is therefore to instead consider the eigenoperators of $\hat{\Gamma}$

$$
\left.\left.\hat{\Gamma} \mid \Psi_{j}\right)=-\zeta_{j} \mid \Psi_{j}\right),
$$

where $\zeta_{j}$ is purely real and non-negative. Eigenoperators $\Psi_{j}^{0}$ with eigenvalue $\zeta_{j}=0$ form the null space, $\operatorname{null}(\hat{\Gamma})$, of the relaxation superoperator ${ }^{43,48,53}$

$$
\left.\left.\hat{\Gamma} \mid \Psi_{j}^{0}\right)=0 \quad \Longleftrightarrow \mid \Psi_{j}^{0}\right) \in \operatorname{null}(\hat{\Gamma}) .
$$


Typically the elements of null( $(\hat{\Gamma})$ are assumed to approximately retain their long-lived behaviour under the action of $\hat{L}$. For example $\Psi_{j}^{0}$ remains an approximate eigenoperator of $\hat{L}$ with its eigenvalue having a vanishing real part

$$
\left.\left.\hat{L} \mid \Psi_{j}^{0}\right) \approx+i \omega_{j}^{\prime} \mid \Psi_{j}^{0}\right)
$$

Generally speaking this is not true and $\hat{L}$ will mix different eigenoperators of $\hat{\Gamma}$. But for the infinite temperature case $\left(\hat{\Gamma}=\hat{\Gamma}^{\dagger}\right)$ we may show that eigenoperator mixing obeys the following rule.

Proposition 1. The eigenoperators $\Phi_{j}^{0}$ of $\hat{L}$ with a vanishing real part may be written as a linear combination of eigenoperators $\Psi_{j}^{0}$ of $\hat{\Gamma}=\hat{\Gamma}^{\dagger}$ with $\zeta_{j}=0$.

Proposition 1 implies that the generic form of the longlived spin operators of $\hat{L}$ is already determined by the structure of $\hat{\Gamma}$, and that their number may never exceed the number of long-lived spin operators of $\hat{\Gamma}$.

Proof. It is sufficient to show that none of the $\Phi_{j}^{0}$ have components along any of the $\Psi_{k}$ with $\zeta_{k}>0$. To see this, consider the following inner product

$$
\begin{aligned}
\left(\Psi_{k}\left|\hat{L}+\hat{L}^{\dagger}\right| \Phi_{j}^{0}\right) & =+i \omega_{j}\left(\Psi_{k} \mid \Phi_{j}^{0}\right)+\left(\Psi_{k} \mid \hat{L}^{\dagger} \Phi_{j}^{0}\right) \\
& =\left(\Psi_{k}\left|\left(-i \hat{H}_{\mathrm{coh}}+\hat{\Gamma}\right)+\left(-i \hat{H}_{\mathrm{coh}}+\hat{\Gamma}\right)^{\dagger}\right| \Phi_{j}^{0}\right) \\
& =2\left(\Psi_{k}|\hat{\Gamma}| \Phi_{j}^{0}\right) \\
& =-2 \zeta_{k}\left(\Psi_{k} \mid \Phi_{j}^{0}\right),
\end{aligned}
$$

To evaluate the action of $\hat{L}^{\dagger}$ onto $\Phi_{j}^{0}$ we consider its interaction with an arbitrary operator $Q$. The operator $Q$ may be decomposed as follows

$$
Q=Q_{\perp}+a \Phi_{j}^{0}
$$

where $Q_{\perp}$ is orthogonal to $\Phi_{j}^{0}$ and $a$ the projection of $Q$ along $\Phi_{j}^{0}$. The inner product of $\left.\hat{L}^{\dagger} \mid \Phi_{j}^{0}\right)$ with $Q$ is then given by

$$
\begin{aligned}
\left(Q \mid \hat{L}^{\dagger} \Phi_{j}^{0}\right) & =\left(\hat{L} Q \mid \Phi_{j}^{0}\right) \\
& =\left(\hat{L} Q_{\perp} \mid \Phi_{j}^{0}\right)+\left(a \hat{L} \Phi_{j}^{0} \mid \Phi_{j}^{0}\right)
\end{aligned}
$$

We utilise the fact that the eigenoperators $\Phi_{j}^{0}$ are ordinary eigenoperators of $\hat{L}$ (see appendix $A$ ). This implies that $\hat{L} Q_{\perp}$ remains orthogonal to $\Phi_{j}^{0}$, leading to the following equality

$$
\begin{aligned}
\left(Q \mid \hat{L}^{\dagger} \Phi_{j}^{0}\right) & =\left(\hat{L} Q_{\perp} \mid \Phi_{j}^{0}\right)+\left(a \hat{L} \Phi_{j}^{0} \mid \Phi_{j}^{0}\right) \\
& =\left(a \hat{L} \Phi_{j}^{0} \mid \Phi_{j}^{0}\right) \\
& =\left(+i \omega_{j} a \Phi_{j}^{0} \mid \Phi_{j}^{0}\right) \\
& =-i \omega_{j}\left(a \Phi_{j}^{0} \mid \Phi_{j}^{0}\right) \\
& =-i \omega_{j}\left(Q_{\perp}+a \Phi_{j}^{0} \mid \Phi_{j}^{0}\right) \\
& =-i \omega_{j}\left(Q \mid \Phi_{j}^{0}\right)
\end{aligned}
$$$$
\text { since }\left(Q_{\perp} \mid \Phi_{j}^{0}\right)=0
$$$$
\text { for } Q \text { arbitrary. }
$$

Replacing $Q$ by $\Psi_{k}$ shows that the first line of equation 22 vanishes, so that the components of $\Phi_{j}^{0}$ along $\Psi_{k}$ are constrained by

$$
\left(\Psi_{k}\left|\hat{L}+\hat{L}^{\dagger}\right| \Phi_{j}^{0}\right)=-2 \zeta_{k}\left(\Psi_{k} \mid \Phi_{j}^{0}\right)=0 .
$$

But since we have assumed that $\zeta_{k}>0$ it follows that $\left(\Psi_{k} \mid \Phi_{j}^{0}\right)=0$ for any $k$.

As shown in appendix D, these arguments are not spoiled by considering relaxation at finite temperature as long as the system fulfills the high-temperature and high-entropy condition. The majority of the remaining discussion we will therefore focus on the characterisation of the null space of $\hat{\Gamma}$ instead of $\hat{L}$.

\section{Lie Algebras}

Unitary evolution of a spin ensemble is generated by a set of hermitian traceless spin operators $\mathcal{Q}$ in the sense that any Hamiltonian $H$ may be represented as a linear combination of the various $Q \in \mathcal{Q}$. We may assign matrix representations to the elements of $\mathcal{Q}$. These may be combined via the matrix commutator $[A, B]=A B-B A$.

Definition II.1. A set of spin operators $\mathcal{Q}$ together with the commutator $[A, B]$ that fulfills the following conditions for any combination of the various $Q$ 's

- Bilinearity: $[a A+b B, C]=a[A, C]+b[B, C]$. $[C, a A+b B]=a[C, A]+b[C, B]$.

- Jacobi identity: $[A,[B, C]]+[C,[A, B]]+[B,[C, A]]=0$.

- Alternativity: $[A, A]=0$.

is called a Lie algebra $\mathcal{L}(\mathcal{Q})^{72,73}$.

A simple example is the set of Cartesian angular momentum operators $\left\{L_{x}, L_{y}, L_{z}\right\}$ with its cyclic commutation relations ${ }^{55-57}$

$$
\left[L_{x}, L_{y}\right]=L_{z}, \quad\left[L_{z}, L_{x}\right]=L_{y}, \quad\left[L_{y}, L_{z}\right]=L_{z}
$$

This set is closed under commutation and fulfills the formal requirements of a Lie algebra.

The set $\mathcal{Q}$ however does not need to exhaust all elements of the corresponding Lie algebra. For example, the set $\left\{L_{x}, L_{y}\right\}$ also generates the Lie algebra of the Cartesian angular momentum operators since we may generate $L_{z}$ through commutation of $L_{x}$ and $L_{y}$. Such a set is called a generating set of the Lie algebra ${ }^{72,73}$. So to be more precise, by $\mathcal{L}(\mathcal{Q})$ we refer to the set of all linearly independent operators generated by all possible nested commutators of the various elements of $\mathcal{Q}$.

The Lie algebra $\mathcal{L}(\mathcal{Q})$ may contain a smaller subset $\mathcal{O}$ that fulfills the conditions of a Lie algebra on its own ${ }^{72,73}$. 
Definition II.2. A subset $\mathcal{O} \subset \mathcal{L}(\mathcal{Q})$ that is closed under commutation

$$
\left[O_{1}, O_{2}\right] \in \mathcal{O},
$$

is called a Lie subalgebra $\mathcal{L}(\mathcal{O}) \subset \mathcal{L}(\mathcal{Q})$.

\section{Centralizer of a Lie Algebra}

An important concept for our characterisation of longlived spin operators is the centralizer $\mathrm{C}(\mathcal{L}(\mathcal{O}))$ of a Lie subalgebra $\mathcal{L}(\mathcal{O}) \subset \mathcal{L}(\mathcal{Q})^{72}$.

Definition II.3. The centralizer $\mathrm{C}(\mathcal{L}(\mathcal{O}))$ is the set of elements $Q \in \mathcal{L}(\mathcal{Q})$ that commute with all elements in $\mathcal{L}(\mathcal{O})$.

$$
\mathrm{C}(\mathcal{L}(\mathcal{O}))=\{Q \in \mathcal{L}(\mathcal{Q}) \mid[Q, O]=0 \forall O \in \mathcal{L}(\mathcal{O})\} .
$$

Additionally, we would like to avoid the necessity of specifying the whole Lie subalgebra $\mathcal{L}(\mathcal{O})$. Instead we would like to work with one of the possible generating sets. In later applications this generating set will typically be determined by the components of the relaxation superoperator $\hat{\Gamma}$. Thankfully the centralizer is completely determined by any of the generating sets $\mathcal{O}$ of $\mathcal{L}(\mathcal{O})$.

Proposition 2. A Lie algebra $\mathcal{L}(\mathcal{O})$ and any of its generating sets $\mathcal{O}$ have the same centralizer, $\mathrm{C}(\mathcal{L}(\mathcal{O}))=\mathrm{C}(\mathcal{O})$.

Proof. This follows from the Jacobi identity, suppose that $C \in \mathrm{C}(\mathcal{O})$ and $O_{1}, O_{2} \in \mathcal{O}$

$$
\begin{aligned}
& {\left[C,\left[O_{1}, O_{2}\right]\right]+\left[O_{2},\left[C, O_{1}\right]\right]+\left[O_{1},\left[O_{2}, C\right]\right]=0} \\
& {\left[C,\left[O_{1}, O_{2}\right]\right]=0 .}
\end{aligned}
$$

Similarly, every possible nested commutator of $O_{1}, O_{2}$ and $C$ vanishes by repeated application of the Jacobi identity. But since $\mathcal{O}$ is a generating set of $\mathcal{L}(\mathcal{O})$ it follows that $\mathrm{C}(\mathcal{O}) \subset \mathrm{C}(\mathcal{L}(\mathcal{O}))$. The inclusion $\mathrm{C}(\mathcal{L}(\mathcal{O})) \subset \mathrm{C}(\mathcal{O})$ follows from the definition of the centralizer.

Consider for example the coherent Hamiltonian given by equation 4 and the set of operators $\{C\}$ that commute with $H_{\text {coh }}$

$$
\left[H_{\mathrm{coh}}, C\right]=\sum_{m} x_{m}\left[X_{m}, C\right]=0 .
$$

The vanishing commutator indicates that the expectation value $\langle C\rangle$ of any operator $C$ remains conserved during the evolution. These expectation values are the constants of motion of the system. But since the coefficients $x_{m}$ are arbitrary, individual commutators must vanish identically

$$
\left[X_{1}, C\right]=0,\left[X_{2}, C\right]=0, \ldots,\left[X_{N}, C\right]=0 .
$$

The question of "Which operators commute with $H_{\text {coh }}$ ?" is therefore equivalent to finding all operators that commute with every element in the set $\left\{X_{m}\right\}$. But this is precisely the centralizer of $\left\{X_{m}\right\}$. The constants of motions are therefore related to the elements of the centralizer

$$
C \in \mathrm{C}\left(\left\{X_{m}\right\}\right) \Longleftrightarrow\langle C\rangle=\text { constant of motion. }
$$

\section{E. Centralizer of the Relaxation Algebra}

According to proposition 1, long-lived spin operators, at least to a first approximation, may be identified as the elements of the null space of $\hat{\Gamma}$. Drawing analogy to the coherent case long-lived spin operators represent "constants of relaxation". It therefore seems reasonable to explore if long-lived spin operators may also be characterised by some type of centralizer.

In order to make that connection more apparent we abbreviate the real part of the spectral densities by

$$
w_{m n}=\operatorname{Re}\left\{J_{m n}\left(\Omega_{n}\right)\right\}
$$

and consider a relaxation superoperator of the form

$$
\hat{\Gamma}=-\sum_{m n} w_{m n} \hat{Y}_{m} \hat{Y}_{n}^{\dagger},
$$

For any valid relaxation superoperator the coefficients $w_{m n}$ form a hermitian positive semidefinite matrix ${ }^{74,75}$

$$
v^{\dagger} W v=\sum_{m n} v_{m}^{*} w_{m n} v_{n} \geq 0 \quad \text { for arbitrary } v,
$$

so that $W$ may be diagonalised. The eigenvalues and eigenvectors of $W$

$$
W z_{k}=d_{k} z_{k}
$$

are used to define a new set of relaxation operators

$$
V_{k}=\left[z_{k}\right]_{m} Y_{m}
$$

This "diagonalised" set of operators $\{V\}$ eliminates the double sum of equation 35

$$
\hat{\Gamma}=-\sum_{k} d_{k} \hat{V}_{k} \hat{V}_{k}^{\dagger}
$$

Motivated by this observation, we define the set of operators $\mathcal{V}=\{V\}$ as the generating set of the relaxation algebra $\mathcal{L}(\mathcal{V})$. We note that if $V$ is not hermitian, then both $V$ and $V^{\dagger}$ are elements of $\mathcal{V}$ since the fluctuating Hamiltonian is hermitian at all points in time.

Theorem II.1 (Centralizer and LLS correspondence). The set of long-lived spin operators $\left\{\Psi_{j}^{0}\right\}$ of $\hat{\Gamma}$ coincides with the centralizer of the relaxation algebra $\mathrm{C}(\mathcal{V})$. 
Proof. Suppose $C \in \mathrm{C}(\mathcal{V})$, since any of the $V_{k}^{\dagger}$ 's is an element of $\mathcal{V}$, the inner commutator of the double commutator vanishes by definition

$$
\hat{V}_{k}^{\dagger} C=\left[V_{k}^{\dagger}, C\right]=0 \quad \forall k .
$$

We therefore have $\mathrm{C}(\mathcal{V}) \subset \operatorname{null}(\hat{\Gamma})$.

Now suppose that $\Psi^{0} \in \operatorname{null}(\hat{\Gamma})$. Due to the hermiticity of $\hat{\Gamma}$ for conjugate pairs $k$ and $k^{*}$ one has

$$
d_{k} \hat{V}_{k} \hat{V}_{k}^{\dagger}+d_{k^{*}} \hat{V}_{k}^{\dagger} \hat{V}_{k}=d_{k}\left(\hat{V}_{k} \hat{V}_{k}^{\dagger}+\hat{V}_{k}^{\dagger} \hat{V}_{k}\right) .
$$

Apart from that the relaxation coefficients $d_{k} \geq 0$ are arbitrary. For any operator $\phi$ and fixed $\Psi^{0}$ one then has

$$
\left(\phi\left|\left(\hat{V}_{k} \hat{V}_{k}^{\dagger}+\hat{V}_{k}^{\dagger} \hat{V}_{k}\right)\right| \Psi^{0}\right)=0 \quad \forall k, \phi .
$$

The particular choice $\phi=\Psi^{0}$ leads to

$$
\begin{aligned}
& \left(\Psi^{0} \mid \hat{V}_{k} \hat{V}_{k}^{\dagger} \Psi^{0}\right)+\left(\Psi^{0} \mid \hat{V}_{k}^{\dagger} \hat{V}_{k} \Psi^{0}\right)=0 \\
& \left(\hat{V}_{k}^{\dagger} \Psi^{0} \mid \hat{V}_{k}^{\dagger} \Psi^{0}\right)+\left(\hat{V}_{k} \Psi^{0} \mid \hat{V}_{k} \Psi^{0}\right)=0 \\
& \left\|\hat{V}_{k}^{\dagger} \Psi^{0}\right\|^{2}+\left\|\hat{V}_{k} \Psi^{0}\right\|^{2}=0 \\
& \left\|\left[V_{k}^{\dagger}, \Psi^{0}\right]\right\|^{2}+\left\|\left[V_{k}, \Psi^{0}\right]\right\|^{2}=0 \quad \forall k .
\end{aligned}
$$

The last equality implies that $\left[V_{k}^{\dagger}, \Psi^{0}\right]=\left[V_{k}, \Psi^{0}\right]=0$ for every $k$. The vanishing commutators on the other hand imply that $\Psi^{0} \in \mathrm{C}(\mathcal{V})$, so that the null space of $\hat{\Gamma}$ is included in the centralizer of the relaxation algebra $\operatorname{null}(\hat{\Gamma}) \subset \mathrm{C}(\mathcal{V})$.

Theorem II.1 represents the main result of our paper establishing a rigorous relation between long-lived spin operators and the centralizer of the relaxation algebra.

The mathematical relationship between long-lived spin operators in NMR and the centralizer concept in Lie Algebra is of conceptual value. Furthermore, the Lie algebra approach to long-lived spin operators leads to an efficient computational method for the identification of long-lived spin operators, as discussed further below.

\section{F. Centralizer for the Symmetric Algebra}

Analytic results for the centralizer of generic relaxation algebra are challenging. But it is well known that longlived spin operators predominantly arise whenever the coherent and fluctuating contributions display some type of internal symmetry ${ }^{43-54}$. The relaxation algebra then consists of a set spin operators invariant under some symmetry group $G$. For such cases it is possible to give an explicit characterisation of the centralizer. As a special case of this we consider spin permutation symmetry.

A spin system displays spin permutation symmetry if its coherent Hamiltonian remains invariant under conjugation by a spin permutation operator $P$

$$
P H_{\mathrm{coh}} P^{\dagger}=H_{\mathrm{coh}} .
$$

Since spin permutations represent unitary operations

$$
P^{\dagger} P=\mathbb{1},
$$

it follows that the set of all such permutations $\{P\}$ forms the permutation group $G_{\mathrm{coh}}=\{P\}$ of the coherent Hamiltonian ${ }^{55,56}$. The Hamiltonian then possesses $G_{\text {coh }}$ symmetry or is invariant under $G_{\text {coh }}$. We will denote such a Hamiltonian by $H_{\text {coh }}^{\star}$ omitting any specific reference to the group $G_{\text {coh }}$.

A simple example is the coherent Hamiltonian for a strongly coupled spin- $1 / 2$ pair in the presence of an external magnetic field

$$
H_{\mathrm{coh}}^{\star}=\omega_{0}\left(I_{1 z}+I_{2 z}\right)+2 \pi J_{12} \mathbf{I}_{1} \cdot \mathbf{I}_{2},
$$

where $\omega_{0}=-2 \pi B_{0} \gamma$ represents the Larmor frequency of the spins and $J_{12}$ their scalar coupling constant. This Hamiltonian is invariant under exchange of spins 1 and 2 . Its symmetry properties may be described by the symmetric group of order two ${ }^{76}$

$$
\mathrm{S}_{2}=\left\{\mathbb{1}, P_{12}\right\} .
$$

Generalisations to larger spin systems are straightfor$\operatorname{ward}^{47,49-51}$.

Similarly, the relaxation superoperator may be invariant under conjugation by a spin permutation superoperator $\hat{P}$

$$
\hat{P} \hat{\Gamma}^{\star} \hat{P}^{\dagger}=\hat{\Gamma}^{\star} .
$$

The collection of all such permutations forms the symmetry group $G_{\hat{\Gamma}}$ of the relaxation superoperator. In general, the symmetry groups $G_{\text {coh }}$ of $H_{\text {coh }}^{\star}$ and $G_{\hat{\Gamma}}$ of $\hat{\Gamma}^{\star}$ are not the same. But in practice it often turns out $G_{\hat{\Gamma}} \subset G_{\text {coh }}$ is a subgroup of the symmetry group of the coherent Hamiltonian $^{50,51}$. A first approximation to the relaxation superoperator is then given by ${ }^{50-53}$

$$
\hat{\Gamma}^{\star}=-\sum_{k} d_{k} \hat{V}_{k}^{\star} \hat{V}_{k}^{\star \dagger}
$$

where the set $\left\{V^{\star}\right\}$ is a collection of symmetric spin operators. We call this set the symmetric relaxation algebra and denote it by $\mathcal{V}^{\star}$.

On the Hilbert space $(\mathbb{H})$ of our system we may assign a matrix representation $D(P)$ to each spin permutation operator $P$. According to standard results from group theory there exists a unitary transformation $T$ that simultaneously block diagonalises all elements of $G_{\hat{\Gamma}}{ }^{55-57}$

$$
T D(P) T^{\dagger}=\bigoplus_{j=1}^{N_{\Gamma}} m_{j}^{\Gamma_{j}} D^{\Gamma_{j}}(P) \quad \forall P \in G_{\hat{\Gamma}} .
$$

The matrices $D^{\Gamma_{j}}(g)$ are the irreducible representations of $G_{\hat{\Gamma}}$ and may be labelled by some index $\Gamma_{j}$. For a given group $G_{\hat{\Gamma}}$ there may exist $N_{\Gamma}$ irreducible representations in total, each representation is of dimensionality 
$d_{j}^{\Gamma}$. Depending on the Hilbert space $\mathbb{H}$ each irreducible representation $\Gamma_{j}$ may occur with a multiplicity $m_{j}^{\Gamma}$. The dimension of $\mathbb{H}$ may therefore be expressed as follows

$$
d_{\mathbb{H}}=\sum_{j=1}^{N_{\Gamma}} m_{j}^{\Gamma} d_{j}^{\Gamma} .
$$

The spin states that generate the irreducible representations of $G_{\hat{\Gamma}}$ form a symmetry adapted basis $\mathcal{B}$. In order to fully specify the basis elements of $\mathcal{B}$ we make use of three "quantum" numbers or group labels

$$
\mathcal{B}=\left\{\left|\Gamma_{j}, \gamma, \mu\right\rangle\right\}
$$

The first label $\Gamma_{j}$ specifies the irreducible representation a spin state belongs to. The second label $\gamma$ distinguishes between basis elements belonging to the same irreducible representation but different "sub-representations" (applies to $d_{j}^{\Gamma}>1$ ). The third label $\mu$ is necessary to distinguish between spin states of identical symmetry $\left(\Gamma_{j}, \gamma\right)$, but belonging to different copies of $\Gamma_{j}$ (applies to $\left.m_{j}^{\Gamma}>1\right)$.

According to the Schur orthogonality relations (see appendix E) transition elements involving symmetric operators $Q^{\star}=P Q^{\star} P^{\dagger}$ and elements of $\mathcal{B}$ obey the following symmetry constraints

$$
\left\langle\Gamma_{j}, \gamma, \mu\left|Q^{\star}\right| \Gamma_{k}, \eta, \nu\right\rangle \propto \delta_{j k} \delta_{\gamma \eta}
$$

Symmetric operators are therefore unable to induce transitions between spin states belonging to different irreducible representations. Within a symmetry manifold symmetric operators additionally preserve the subrepresentation of a given spin state.

Equation 53 indicates that the matrix representation $D\left(V^{\star}\right)$ of any operator of the relaxation algebra $\mathcal{V}^{\star}$ splits into the following components

$$
\begin{aligned}
& D\left(V^{\star}\right)=\underbrace{K^{\Gamma_{1}}\left(V^{\star}\right) \oplus \cdots \oplus K^{\Gamma_{1}}\left(V^{\star}\right)}_{d_{1}^{\Gamma}} \oplus \cdots \\
& \oplus \underbrace{K^{\Gamma_{N_{\Gamma}}}\left(V^{\star}\right) \oplus \cdots \oplus K^{\Gamma_{N_{\Gamma}}\left(V^{\star}\right)}}_{d_{N_{\Gamma}}^{\Gamma}},
\end{aligned}
$$

where each $K^{\Gamma_{j}}$ is of dimension $m_{j}^{\Gamma} \times m_{j}^{\Gamma}$. We deliberately chose the symbol $K^{\Gamma_{j}}$ to distinguish these matrices from the irreducible representations $D^{\Gamma_{j}}$.

The centralizer $\mathrm{C}\left(\mathcal{V}^{\star}\right)$ may now be determined by explicitly considering the resulting commutator relations. For simplicity we consider an irreducible representation $\Gamma_{k}$ with dimensionality $d_{k}^{\Gamma}=2$ and multiplicity $m_{k}^{\Gamma}$. The commutator in block diagonal matrix form reads as follows

$$
\begin{aligned}
& {\left[\begin{array}{cc}
K^{\Gamma_{k}}\left(V^{\star}\right) & 0 \\
0 & K^{\Gamma_{k}}\left(V^{\star}\right)
\end{array}\right]\left[\begin{array}{cc}
A & B \\
C & D
\end{array}\right]-} \\
& {\left[\begin{array}{cc}
A & B \\
C & D
\end{array}\right]\left[\begin{array}{cc}
K^{\Gamma_{k}}\left(V^{\star}\right) & 0 \\
0 & K^{\Gamma_{k}}\left(V^{\star}\right)
\end{array}\right]=0 .}
\end{aligned}
$$

Carrying out block matrix multiplication leads to the following set of equations

$$
\left[\begin{array}{ll}
{\left[K^{\Gamma_{k}}\left(V^{\star}\right), A\right]} & {\left[K^{\Gamma_{k}}\left(V^{\star}\right), B\right]} \\
{\left[K^{\Gamma_{k}}\left(V^{\star}\right), C\right]} & {\left[K^{\Gamma_{k}}\left(V^{\star}\right), D\right]}
\end{array}\right]=0 .
$$

If the symmetric relaxation algebra exhausts the set of all symmetric operators, then the only matrix that fulfils these conditions for every $V^{\star} \in \mathcal{V}^{\star}$ is given by

$$
\begin{aligned}
{\left[\begin{array}{ll}
A & B \\
C & D
\end{array}\right]=\left[\begin{array}{ll}
a \mathbb{1}_{m_{k}^{\Gamma}} & b \mathbb{1}_{m_{k}^{\Gamma}} \\
c \mathbb{1}_{m_{k}^{\Gamma}} & d \mathbb{1}_{m_{k}^{\Gamma}}
\end{array}\right] } & =\left[\begin{array}{ll}
a & b \\
c & d
\end{array}\right] \otimes \mathbb{1}_{m_{k}^{\Gamma}} \\
& =M_{d_{k}^{\Gamma}} \otimes \mathbb{1}_{m_{k}^{\Gamma}},
\end{aligned}
$$

where $\mathbb{1}_{m_{k}^{\Gamma}}$ represents an $m_{k}^{\Gamma} \times m_{k}^{\Gamma}$ dimensional identity matrix and $M_{d_{k}^{\Gamma}}$ a generic $d_{k}^{\Gamma} \times d_{k}^{\Gamma}$ dimensional matrix.

A similar analysis is applicable to any other irreducible representation $\Gamma_{j}$. The centralizer $\mathrm{C}\left(\mathcal{V}^{\star}\right)$ of the symmetric relaxation algebra is thus given by

$$
\mathrm{C}\left(\mathcal{V}^{\star}\right)=\bigoplus_{j=1}^{N_{\Gamma}} M_{d_{j}^{\Gamma}} \otimes \mathbb{1}_{m_{j}^{\Gamma}}
$$

(Although different in interpretation, these matrices resemble the center of the symmetric group ${ }^{76}$.)

From equation 58 it follows that the number of longlived spin operators is given by

$$
N_{\mathrm{LLS}}=\sum_{j=1}^{N_{\Gamma}} d_{j}^{\Gamma} \times d_{j}^{\Gamma} .
$$

This number may be split into the number of long-lived spin populations and coherences

$$
\begin{aligned}
N_{\mathrm{LLS}}^{\mathrm{P}} & =\sum_{j=1}^{N_{\Gamma}} d_{j}^{\Gamma} \\
N_{\mathrm{LLS}}^{\mathrm{C}} & =\sum_{j=1}^{N_{\Gamma}} d_{j}^{\Gamma} \times\left(d_{j}^{\Gamma}-1\right) .
\end{aligned}
$$

This result agrees with our previous analysis on the number of long-lived spin operators based on symmetry projection operator techniques ${ }^{53}$. This is not too surprising, expressing the symmetry projection operators in terms of symmetry adapted spin states, it is easy to verify that the elements of the centralizer $\mathrm{C}\left(\mathcal{V}^{\star}\right)$ span the same operator space as the corresponding symmetry projection operators.

\section{G. Centralizer for a generic Algebra}

In some cases the symmetric relaxation algebra $\mathcal{V}^{\star}$ displays symmetry constraints beyond permutation symmetry $31,32,52,53$. An example of such a case would be a rapidly "rotating" methyl group dominated by dipolar relaxation. The rapid methyl rotation imposes $\mathrm{S}_{3}$ 
symmetry onto the relaxation superoperator, but the assumption of predominately dipolar relaxation further restricts the symmetric relaxation algebra $\mathcal{V}^{\star}$ to irreducible tensor operators of spherical rank $k=2$.

For $\mathrm{CH}_{3}$ groups a full characterisation of its long-lived operators may be given by utilising the Schur-Weyl duality theorem ${ }^{53}$. In more general cases however, such as rapidly reorienting $\mathrm{CD}_{3}$ for example, an analysis based on rotational-permutational dual-pairings becomes increasingly difficult and has only been partially resolved ${ }^{53,54}$.

To calculate the centralizer for a general relaxation algebra we note the following relation between the centralizer of the combined Lie algebra $\mathcal{L}\left(\mathcal{O}_{a} \cup \mathcal{O}_{b}\right)$ and its constituents $\mathcal{L}\left(\mathcal{O}_{a}\right), \mathcal{L}\left(\mathcal{O}_{b}\right)$.

Proposition 3. The centralizer for the combined Lie algebra $\mathcal{L}\left(\mathcal{O}_{a} \cup \mathcal{O}_{b}\right)$ is given by $\mathrm{C}\left(\mathcal{O}_{a} \cup \mathcal{O}_{b}\right)=\mathrm{C}\left(\mathcal{O}_{a}\right) \cap \mathrm{C}\left(\mathcal{O}_{b}\right)$.

Proof. Consider an element $C \in \mathrm{C}\left(\mathcal{O}_{a}\right) \cap \mathrm{C}\left(\mathcal{O}_{b}\right)$ and $O_{12} \in \mathcal{L}\left(\mathcal{O}_{a} \cup \mathcal{O}_{b}\right)$. If the operator $O_{12}$ is an element of $\mathcal{L}\left(\mathcal{O}_{a}\right)$ or $\mathcal{L}\left(\mathcal{O}_{b}\right)$ it commutes with $C$ by definition, otherwise $\mathrm{O}_{12}$ is the result of a (possibly) nested commutator. Repeated application of the Jacobi identity shows that $\left[C, O_{12}\right]=0$, this implies that $\mathrm{C}\left(\mathcal{O}_{a}\right) \cap \mathrm{C}\left(\mathcal{O}_{b}\right) \subset$ $\mathrm{C}\left(\mathcal{O}_{a} \cup \mathcal{O}_{b}\right)$. The inclusion $\mathrm{C}\left(\mathcal{O}_{a} \cup \mathcal{O}_{b}\right) \subset \mathrm{C}\left(\mathcal{O}_{a}\right) \cap \mathrm{C}\left(\mathcal{O}_{b}\right)$ follows from the fact that $\mathcal{L}\left(\mathcal{O}_{a}\right), \mathcal{L}\left(\mathcal{O}_{b}\right) \subset \mathcal{L}\left(\mathcal{O}_{a} \cup \mathcal{O}_{b}\right)$ and the definition of the centralizer.

If we consider elements $V_{k} \in \mathcal{V}$ of the relaxation algebra on their own, then repeated application of proposition 3 shows that the centralizer $\mathrm{C}(\mathcal{V})$ is given by a pair-wise intersection of the null spaces null $\left(\hat{V}_{k}\right)$

$$
\mathrm{C}(\mathcal{V})=\operatorname{null}\left(\hat{V}_{1}\right) \cap \operatorname{null}\left(\hat{V}_{2}\right) \cap \cdots \cap \operatorname{null}\left(\hat{V}_{N}\right)
$$

The calculation of all long-lived spin operators is therefore reduced to a simple linear algebra problem. A particularly simple procedure for the calculation of a general centralizer is given by Algorithm 1.

\section{Algorithm 1: Centralizer for a relaxation} algebra $\mathcal{V}$.

\footnotetext{
function Centralizer $(\mathcal{V})$;

Input : A generating set of matrices for the relaxation algebra $\mathcal{L}(\mathcal{V})$

Output: $\mathrm{C}(\mathcal{V})$

for $i=1 ; i \leq N$ do

$\hat{V}_{i}=V_{i} \otimes \mathbb{1}-\mathbb{1} \otimes V_{i}^{\mathrm{T}}$

end

Centralizer $=\operatorname{null}\left(\hat{V}_{1}\right)$

for $i=2 ; i \leq N$ do

Centralizer $=$ Centralizer $\cap \operatorname{null}\left(\hat{V}_{i}\right)$

end

return Centralizer
}

\section{ILLUSTRATIVE NMR EXAMPLES}

\section{A. Spin-1/2 pairs}

\section{Isotropic random fields}

The simplest non-trivial long-lived spin operator may be found in coupled spin-1/2 pairs. Consider for example a coupled spin- $1 / 2$ pair at sufficiently low magnetic fields. The coherent Hamiltonian is then given by

$$
H_{\mathrm{coh}}^{\star}=2 \pi J_{12} \mathbf{I}_{1} \cdot \mathbf{I}_{2} \text {. }
$$

The star indicates $\mathrm{S}_{2}$ spin permutation symmetry of the Hamiltonian.

Let us further assume that the relaxation process is driven by perfectly correlated random fields acting symmetrically on spins 1 and 2. The fluctuating Hamiltonian is then also $\mathrm{S}_{2}$ symmetric and given by

$$
H_{\text {fluc }}^{\star}(t)=\sum_{\mu \in\{x, y, z\}} \omega_{\mu}(t)\left(I_{1 \mu}+I_{2 \mu}\right)=\sum_{\mu \in\{x, y, z\}} \omega_{\mu}(t) I_{\mu} .
$$

Following equation 12 the resulting relaxation superoperator may be expressed as follows

$$
\begin{aligned}
\hat{\Gamma}^{\star} & =-\sum_{\mu, \mu^{\prime} \in\{x, y, z\}} \int_{0}^{+\infty} \overline{\omega_{\mu}(0) \omega_{\mu^{\prime}}^{*}(\tau)} \hat{I}_{\mu} \hat{I}_{\mu^{\prime}}^{\dagger} d \tau \\
& =-\sum_{\mu, \mu^{\prime} \in\{x, y, z\}} \int_{0}^{+\infty} k_{\mu \mu^{\prime}}(\tau) \hat{I}_{\mu} \hat{I}_{\mu^{\prime}}^{\dagger} d \tau \\
& =-\sum_{\mu, \mu^{\prime} \in\{x, y, z\}} w_{\mu \mu^{\prime}} \hat{I}_{\mu} \hat{I}_{\mu^{\prime}}^{\dagger}
\end{aligned}
$$

The matrix $W$ may be identified as a collection of crosscorrelation integrals

$$
W=\int_{0}^{+\infty}\left[\begin{array}{lll}
k_{x x}(\tau) & k_{x y}(\tau) & k_{x z}(\tau) \\
k_{x y}(\tau) & k_{y y}(\tau) & k_{y z}(\tau) \\
k_{x z}(\tau) & k_{y z}(\tau) & k_{z z}(\tau)
\end{array}\right] d \tau
$$

By definition $W$ is symmetric $W=W^{\dagger}$ and positive semidefinite $W \geq 0$.

If the fluctuations are additionally spatially isotropic $W$ may be expressed as follows ${ }^{43}$

$$
W=\tau_{\text {rand }}\left(\gamma B_{\text {rand }}^{\text {rms }}\right)^{2}\left[\begin{array}{lll}
1 & \xi & \xi \\
\xi & 1 & \xi \\
\xi & \xi & 1
\end{array}\right]
$$

Here, $\tau_{\text {rand }}$ and $B_{\text {rand }}^{\text {rms }}$ describe the correlation time and amplitude of the fluctuations, whereas $0 \leq \xi \leq 1$ describes the correlations between fluctuations along two orthogonal axes.

The matrix $W$ is readily orthogonalised, its eigenvalues 
and eigenvectors are given by

$$
\left[\begin{array}{c|c|c}
d_{1} & d_{2} & d_{3} \\
\hline z_{1} & z_{1} & z_{2}
\end{array}\right]=\left[\begin{array}{c|c|c}
1+2 \xi & 1-\xi & 1-\xi \\
\hline \frac{1}{\sqrt{3}} & -\frac{1}{\sqrt{6}} & -\frac{1}{\sqrt{2}} \\
\frac{1}{\sqrt{3}} & \sqrt{\frac{2}{3}} & 0 \\
\frac{1}{\sqrt{3}} & -\frac{1}{\sqrt{6}} & \frac{1}{\sqrt{2}}
\end{array}\right] .
$$

Following equation 38 the generating set $\mathcal{V}^{\star}$ of the symmetric relaxation algebra $\mathcal{L}\left(\mathcal{V}^{\star}\right)$ is given by

$\mathcal{V}^{\star}=\left\{\frac{1}{\sqrt{3}}\left(I_{x}+I_{y}+I_{z}\right), \frac{1}{\sqrt{6}}\left(2 I_{y}-I_{x}-I_{z}\right), \frac{1}{\sqrt{2}}\left(I_{z}-I_{x}\right)\right\}$.

Since any $V \in \mathcal{V}^{\star}$ is invariant under elements of the symmetric group $\mathrm{S}_{2}$, it is simple enough to calculate the centralizer $\mathrm{C}\left(\mathcal{V}^{\star}\right)$ analytically. The symmetric group $\mathrm{S}_{2}$ is characterised by its gerade $(g)$ and ungerade (u) irreducible representations. Both $g$ and $u$ are onedimensional representations so that according to equation 59 the centralizer $\mathrm{C}\left(\mathcal{V}^{\star}\right)$ is two-dimensional. As may be verified by direct calculation, the centralizer elements are the projectors $\left(\Pi_{\Gamma}\right)$ onto the different symmetry manifolds

$$
\mathrm{C}\left(\mathcal{V}^{\star}\right)=\left\{\Pi_{g}, \Pi_{u}\right\}=\left\{\mathbb{1}+P_{12}, \mathbb{1}-P_{12}\right\} .
$$

Choosing $\Pi_{g}$ and $\Pi_{u}$ to be orthonormal with respect to the trace inner product transforms the centralizer $\mathrm{C}\left(\mathcal{V}^{\star}\right)$ into a more familiar form

$$
\mathrm{C}\left(\mathcal{V}^{\star}\right)=\left\{\frac{1}{2} \mathbb{1}, \frac{2}{\sqrt{3}} \mathbf{I}_{1} \cdot \mathbf{I}_{2}\right\} .
$$

The first element represents the conservation of all populations as required by any reasonable physical process. The second centralizer element is the well-known singlet order operator representing a population difference across the $(g, u)$ symmetry manifolds of the system ${ }^{77,78}$.

\section{Anti-correlated random fields}

We now consider a slightly less intuitive example of random field relaxation. We leave the coherent Hamiltonian unchanged, but instead consider the following fluctuating Hamiltonian

$$
H_{\text {fluc }}(t)=\omega_{z}(t)\left(I_{1 z}+I_{2 z}\right)+\sum_{\mu \in\{x, y\}} \omega_{\mu}(t)\left(I_{1 \mu}-I_{2 \mu}\right) .
$$

The two spins experience identical fluctuations along the $z$-axis, but fluctuations along the $x$-and $y$-axis are perfectly anti-correlated.

The fluctuating Hamiltonian 71 does not possess any definite symmetry and is in fact a mixture of symmetric and anti-symmetric contributions. As a result typical symmetry arguments would either lead to a) the conclusion that no long-lived spin operator exists, or $\mathbf{b}$ ) at best acknowledge the possibility of a long-lived spin operator without explicit means of calculation. The centralizer approach on the other hand gives a definite answer.

Taking $W$ to equal the cross-correlation matrix of equation 66 the generating set of the relaxation algebra $\mathcal{V}$ is given by

$$
\begin{aligned}
\mathcal{V}=\{ & \frac{1}{\sqrt{3}}\left(\left(I_{1 x}-I_{2 x}\right)+\left(I_{1 y}-I_{2 y}\right)+\left(I_{1 z}+I_{2 z}\right)\right), \\
& \frac{1}{\sqrt{6}}\left(2\left(I_{1 y}-I_{2 y}\right)-\left(I_{1 x}-I_{2 x}\right)-\left(I_{1 z}+I_{2 z}\right)\right), \\
& \left.\frac{1}{\sqrt{2}}\left(\left(I_{1 z}+I_{2 z}\right)-\left(I_{1 x}-I_{2 x}\right)\right)\right\} .
\end{aligned}
$$

An implementation of algorithm 1 then returns the following centralizer

$$
\begin{aligned}
\mathrm{C}(\mathcal{V}) & =\left\{C_{1}, C_{2}\right\} \\
& =\left\{\frac{1}{2} \mathbb{1}, \frac{2}{\sqrt{3}}\left(I_{1 x} \cdot I_{2 x}+I_{1 y} \cdot I_{2 y}-I_{1 z} \cdot I_{2 z}\right)\right\} .
\end{aligned}
$$

In contrast to conventional symmetry arguments, the centralizer approach does indeed return one non-trivial long-lived spin operator. This operator is clearly not given by pure singlet order, which is a consequence of the symmetry breaking fluctuating Hamiltonian. Little thought however shows that $C_{2}$ is a mixture of singlet order and dipolar order.

\section{B. Methyl rotor}

Long-lived spin operators in rapidly rotating methyl groups are of great practical relevance, due to their connection to quantum rotor induced polarisation (QRIP) effects, for example $31-37,52,54,79,80$. Extensive discussions of long-lived spin operators in rotating methyl groups have been given in references $52-54$. However, as previously mentioned, a complete characterisation of long-lived spin operators in rotating methyl groups requires careful consideration of rotational-permutational dual symmetries. As we will show now, this is in stark contrast to the simplicity of the centralizer approach.

Consider a $\mathrm{CH}_{3}$ methyl rotor consisting of three coupled spin- $1 / 2$ particles. The coherent Hamiltonian is given by

$$
H_{\mathrm{coh}}^{\star}=\omega_{0}\left(I_{1 z}+I_{2 z}+I_{3 z}\right)+2 \pi J_{\mathrm{HH}} \sum_{i<j}^{3} \mathbf{I}_{i} \cdot \mathbf{I}_{j} .
$$

Due to the three-fold symmetry axis of the methyl rotor the coherent spin Hamiltonian displays $\mathrm{S}_{3}$ spin permutation symmetry.

If the methyl group is rotating sufficiently fast, the fluctuating Hamiltonian is subjected to dynamical averaging effects. These dynamical averaging effects impose approximate $\mathrm{S}_{3}$ symmetry onto the fluctuating Hamiltonian. We further assume that spin interactions with 
spherical rank $k \neq 2$ do not significantly contribute to the relaxation process. In this case the fluctuating Hamiltonian for a rapidly rotating methyl group is dominated by dipolar relaxation mechanisms

$$
H_{\text {fluc }}^{\star}(t)=\sum_{m=-2}^{2} y_{m}(t) \sum_{i<j}^{3} T_{2 m}^{i j} .
$$

The operators $T_{2 m}^{i j}$ are irreducible spherical tensor operators resulting from angular momentum coupling of spins $i$ and $j$ (see appendix F). The fact that the fluctuating coefficients $y_{m}(t)$ are independent of the indices $(i, j)$ reflects the $\mathrm{S}_{3}$ symmetry of the problem.

At sufficiently high magnetic fields one may apply the so-called secular approximation to $\hat{\Gamma}^{4-7}$. The dipolar relaxation superoperator for a rapidly rotating methyl group may then be expressed as follows ${ }^{52,53}$

$$
\hat{\Gamma}^{\star}=-\sum_{m=-2}^{2} w_{m m}\left(\sum_{i<j}^{3} \hat{T}_{2 m}^{i j}\right)\left(\sum_{i<j}^{3} \hat{T}_{2 m}^{i j}\right)^{\dagger}
$$

with

$$
w_{m m}=\int_{0}^{+\infty} \overline{y_{m}(0) y_{m}^{*}(\tau)} \cos \left(m \omega_{0} \tau\right) d \tau .
$$

The secularization procedure essentially removes all contributions $w_{m n}$ with $m \neq n$. As a result there is no need to diagonalise the relaxation operators and the generating set of the relaxation algebra consists of symmetrised dipolar interaction terms

$$
\mathcal{V}^{\star}=\left\{\sum_{i<j}^{3} T_{2-2}^{i j}, \sum_{i<j}^{3} T_{2-1}^{i j}, \sum_{i<j}^{3} T_{20}^{i j}, \sum_{i<j}^{3} T_{2+1}^{i j}, \sum_{i<j}^{3} T_{2+2}^{i j}\right\} .
$$

This relaxation algebra displays dual symmetry. Firstly the elements of $\mathcal{V}^{\star}$ are invariant under conjugation by elements of the symmetric group $\mathrm{S}_{3}$, but secondly the restriction to spherical interactions with $k=2$ imposes rotational symmetry onto $\mathcal{V}^{\star}$.

A calculation of the corresponding centralizer $\mathrm{C}\left(\mathcal{V}^{\star}\right)$ with algorithm 1 returns several long-lived spin operators. When expressed in terms of $\mathrm{S}_{3}$ symmetry adapted basis states (see appendix $\mathrm{G}$ ) the centralizer $\mathrm{C}\left(\mathcal{V}^{\star}\right)$ takes the form

$$
\mathrm{C}\left(\mathcal{V}^{\star}\right)=\left[\begin{array}{cccccccc}
c_{1} & 0 & 0 & 0 & 0 & 0 & 0 & 0 \\
0 & c_{1} & 0 & 0 & 0 & 0 & 0 & 0 \\
0 & 0 & c_{1} & 0 & 0 & 0 & 0 & 0 \\
0 & 0 & 0 & c_{1} & 0 & 0 & 0 & 0 \\
0 & 0 & 0 & 0 & c_{2} & c_{3} & c_{4} & c_{5} \\
0 & 0 & 0 & 0 & c_{6} & c_{7} & c_{8} & c_{9} \\
0 & 0 & 0 & 0 & c_{10} & c_{11} & c_{12} & c_{13} \\
0 & 0 & 0 & 0 & c_{14} & c_{15} & c_{16} & c_{17}
\end{array}\right]
$$

as may be verified by direct calculation. The number of independent long-lived spin operators is therefore given by $N_{\text {LLS }}=17$.
To the best of our knowledge, such an increase in the number of long-lived spin operators of rapidly rotating methyl groups has first been noted by Stevanato (personal communication). Following reference 53 a physical explanation of this effect may be given in terms of rotational and permutational dual symmetries. According to the Schur-Weyl duality theorem there exists a one-toone correspondence between irreducible representations of the symmetric group $\mathrm{S}_{N}$ and irreducible representations of the unitary group $\mathrm{U}(n)^{81,82}$. This implies that for a protonated methyl rotor we may always choose a set of symmetry adapted spin states with definite $\mathrm{S}_{3}$ permutation symmetry $\Gamma_{j}$ and total angular momentum $I$. The rotational permutational pairing for these states may be expressed as follows

$$
4 \times\left(\mathrm{A}_{1}, 3 / 2\right) \oplus 4 \times(\mathrm{E}, 1 / 2),
$$

where $\mathrm{A}_{1}$ refers to the trivial and $\mathrm{E}$ to the standard irreducible representation of $\mathrm{S}_{3}{ }^{76}$. Transition elements involving the fluctuating Hamiltonian of equation 75 and any of the dual symmetry adapted spin states are therefore not only constrained by the Schur orthogonality relations, but also by the Wigner-Eckart theorem. One may then show that matrix elements involving the E states and $H_{\text {fluc }}^{\star}(t)$ vanish

$$
\begin{aligned}
& \left\langle\mathrm{E}, 1 / 2, m_{1}\left|H_{\text {fluc }}^{\star}(t)\right| \mathrm{E}, 1 / 2, m_{2}\right\rangle= \\
& \sum_{m=-2}^{2} y_{m}(t) \sum_{i<j}^{3}\left\langle\mathrm{E}, 1 / 2, m_{1}\left|T_{2 m}^{i j}\right| \mathrm{E}, 1 / 2, m_{2}\right\rangle=0 .
\end{aligned}
$$

The $4 \times 4=16$ operators of the E symmetry manifold are therefore immune to dipolar relaxation. This property accounts for the unusual increase in the number of longlived spin operators.

As should be apparent from the discussion above an extension of dual symmetry arguments to generic spin systems can be a challenging process. The centralizer approach on the other hand does not require any sophisticated dual symmetry arguments to arrive at identical results. Instead dual symmetries are naturally incorporated into the calculation process. All it takes is an application of algorithm 1 to the (dual) symmetric relaxation algebra of equation 78 .

\section{SUMMARY}

Nuclear long-lived spin operators represent an integral part of modern NMR. At first, collective efforts largely explored and exploited the various relaxation properties of long-lived spin operators ${ }^{9-13,44-46,83-85}$. Nowadays however applications of nuclear long-lived spin operators go well beyond that with noticeable impact on NMR imaging techniques, zero- to ultralowfield (ZULF) NMR and nuclear hyperpolarisation techniques $^{14-18,86-100}$. But despite a multitude of long-lived 
operators applications, the screening procedure for longlived spin operators has changed little over the years and still relies on tricky group theoretical arguments.

In this work we have presented a fundamentally different approach to the calculation of long-lived spin operators. The concept of a relaxation algebra enabled us to apply Lie algebraic methods to the characterisation of long-lived spin operators. The relaxation algebra itself is generated by the set of diagonalised relaxation operators of $\hat{\Gamma}$. We have established a rigorous connection between the centralizer of the relaxation algebra and the set of long-lived spin operators. For practical purposes we have outlined a simple algorithm for its calculation. In contrast to group theoretical methods however, the centralizer approach does not require any intricate symmetry arguments and several advantages have been illustrated by considering some simple example cases.

Due to its striking simplicity and straightforward automation we believe that the centralizer approach represents a powerful tool in the relaxation analysis of spin operators and other related fields. For example, the study of non-decaying quantum equilibrium correlations has recently sparked some interest ${ }^{101-104}$. The work by Uhrig et al. ${ }^{102}$ for example derived a generalisation of Mazur's inequality setting a lower bound on the amplitude of persisting quantum correlations ${ }^{105,106}$. Their approach relies on several important assumptions, most importantly the ability to fully specify the system-environment interaction and the ability to specify the constants of motion of the system. For several practical situations this is impossible due to the complexity of the environment. As a result the dynamics of a system in contact with a thermal environment are described by a quantum master equation. Since long-lived spin operators may qualitatively be interpreted as constants of relaxation of such master equations, it seems reasonable to expect that similar statements about persisting quantum correlations may be made by replacing Hamiltonian constants of motion with long-lived spin operators.

In the future we therefore hope to incorporate a full implementation of the centralizer approach into our Mathematica ${ }^{107}$ based software package SpinDynamica ${ }^{108}$ and extend its capabilities to arbitrarily thermalised spin systems.

\section{ACKNOWLEDGMENTS}

I thank Malcolm H. Levitt for fruitful discussions and critical comments to the manuscript. This research was funded by the European Research Council (786707FunMagResBeacons), and the Engineering and Physical Sciences Research Council (grants EP/P009980/1 and $\mathrm{EP} / \mathrm{P} 030491 / 1)$.

\section{DATA AVAILABILITY}

The data that support the findings of this study are available from the corresponding author upon reasonable request.

\section{Appendix A: Ordinary eigenoperators of the Liouvillian}

Lemma A.1. Eigenoperators of the Liouvillian $\hat{L}$ corresponding to eigenvalues with a vanishing real part are ordinary eigenoperators.

Proof. Take $X$ to be a matrix with generalised right eigenoperators $\mid \Psi_{j}$ ) of $\hat{L}$ as its columns. The matrix $X$ transforms $\hat{L}$ into Jordan canonical form

$$
X^{-1} \hat{L} X=J=\bigoplus_{i=1}^{M} J_{i}
$$

Each $J_{i}$ represents a Jordan block

$$
J_{i}=\left[\begin{array}{cccc}
\Lambda_{i} & 1 & & \\
& \Lambda_{i} & \ddots & \\
& & \ddots & 1 \\
& & & \Lambda_{i}
\end{array}\right]=\Lambda_{i} \mathbb{1}+N_{i}
$$

where $N_{i}$ is nilpotent $\left(N_{i}^{k}=0\right)$ for some integer $k$. The order of the eigenvalues is arbitrary and we may set $\Lambda_{1}=0$. For infinite temperature we further have

$$
(Q|\hat{L}| \mathbb{1})=0 \quad \forall Q
$$

The identity is disconnected from all other elements and we may set $J_{M}=0$ with $\left.\mid \Psi_{N_{\mathbb{L}}}\right)=\mid \mathbb{1}$ ) as the corresponding eigenoperator.

Suppose now that the Jordan block $J_{1}$ is not diagonal, for example

$$
J_{1}=\left[\begin{array}{cccc}
0 & 1 & & \\
& 0 & \ddots & \\
& & \ddots & 1 \\
& & & 0
\end{array}\right]=N_{1}
$$

The evolution generated by $J_{1}$ is given by

$$
\exp \left(J_{1} t\right)=\sum_{m=0}^{k-1} \frac{t^{m}}{m !} N_{0}^{m}=\left[\begin{array}{ccccc}
1 & t & \frac{t^{2}}{2} & \cdots & \frac{t^{k-1}}{(k-1) !} \\
& \ddots & \ddots & \ddots & \vdots \\
& & 1 & t & \frac{t^{2}}{2} \\
& & & 1 & t \\
& & & & 1
\end{array}\right] .
$$

Without loss of generality we may assume that $\left.\left.X \mid e_{1}\right)=\mid \Psi_{1}^{0}\right)$ is hermitian. If this is not the case the adjoint $\left.\mid \Psi_{1}^{0 \dagger}\right)$ must follow the dynamics of $\left.\mid \Psi_{1}^{0}\right)$ since $\hat{L}$ is hermiticity preserving and the following arguments may 
easily be adjusted. Choose the initial density operator to be given by

$$
\left.\left.\mid \rho(0))=N_{\mathbb{H}}^{-1} \mid \mathbb{1}\right)+c(0) \mid \Psi_{1}^{0}\right) .
$$

The initial condition $c(0)$ is chosen so that $\rho(0)$ represents a valid probability distribution. This is always possible since $\Psi_{1}^{0}$ is hermitian. For example one may choose

$$
c(0)=\left(N_{\mathbb{H}} \sigma_{\max }\right)^{-1},
$$

where $\sigma_{\max }$ is the largest singular value of $\Psi_{1}^{0}$. The evolution of $\rho(0)$ is then given by

$$
\begin{aligned}
\mid \rho(t)) & =\exp (\hat{L} t) \mid \rho(0)) \\
& \left.\left.\left.=N_{\mathbb{H}}^{-1} \mid \mathbb{1}\right)+c(0) \mid \Psi_{1}^{0}\right)+c(0) \mid \Psi_{2}^{0}\right) t .
\end{aligned}
$$

But since $\left(\mathbb{1} \mid \Psi_{2}^{0}\right)=0$ the operator $\Psi_{2}^{0}$ is traceless and must possess at least one negative eigenvalue $\chi<0$. The probability of observing the eigenstate $|\chi\rangle$ is given by

$$
\begin{aligned}
p_{|\chi\rangle}(t) & =\langle\chi|\rho(t)| \chi\rangle \\
& =N_{\mathbb{H}}^{-1}+c(0)\left\langle\chi\left|\Psi_{1}^{0}\right| \chi\right\rangle-|\chi| c(0) t .
\end{aligned}
$$

Clearly for sufficiently long times $t$ the probability $p_{|\chi\rangle}(t)$ attains negative values. The Jordan block $J_{1}$ would therefore break the positivity of $\rho(t)$ unless the first row of $J_{1}$ equals 0

$$
J_{1}=\left[\begin{array}{ccccc}
0 & & \cdots & & 0 \\
& 0 & 1 & & \\
\vdots & & 0 & \ddots & \vdots \\
& & \ddots & 1 \\
0 & & & 0
\end{array}\right] .
$$

But this implies that $\mid \Psi_{1}^{0}$ ) represents the ordinary eigenoperator

$$
\left.\left.\hat{L} \mid \Psi_{1}^{0}\right)=0 \times \mid \Psi_{1}^{0}\right)=0 .
$$

Continuing in this fashion leads to the conclusion that $J_{1}$ is given by

$$
J_{1}=\operatorname{diag}(0,0, \cdots, 0,0) .
$$

This means that every eigenoperator belonging to the $J_{1}$ Jordan block represents an ordinary eigenoperator. A similar analysis may be applied to Jordan blocks $J_{n}$ with $\Lambda_{n}=-i \omega_{n}$ and their corresponding conjugate block $J_{n^{*}}$ by observing the density operator stroboscopically at times $t_{m}=2 \pi m / \omega_{n}$.

\section{Appendix B: Semi-classical master equation}

As described in section II A the fluctuating contributions are in general different for each ensemble member at a given point in time. Consider therefore the equation of motion of a single ensemble member as described within the interaction frame of the coherent Liouvillian

$$
\left.\left.\frac{d}{d t} \mid \tilde{\sigma}(t)\right)=\tilde{\hat{L}}_{\text {fluc }}(t) \mid \tilde{\sigma}(t)\right) .
$$

Here, $\sigma(t)$ describes the density operator of a single ensemble member and the tilde indicates the interaction frame transformation defined by

$$
\begin{aligned}
& \left.\mid \tilde{\sigma}(t))=\exp \left\{-\hat{L}_{\mathrm{coh}} t\right\} \mid \sigma(t)\right), \\
& \tilde{\hat{L}}_{\text {fluc }}(t)=\exp \left\{-\hat{L}_{\mathrm{coh}} t\right\} \hat{L}_{\text {fluc }}(t) \exp \left\{+\hat{L}_{\mathrm{coh}} t\right\} .
\end{aligned}
$$

Following standard NMR literature (see references $4-7$ for example) equation B1 up to second order in timedependent perturbation theory is given by

$$
\left.\left.\left.\frac{d}{d t} \mid \tilde{\sigma}(t)\right)=\tilde{\hat{L}}_{\text {fluc }}(t) \mid \tilde{\sigma}(0)\right)+\int_{0}^{t} \tilde{\hat{L}}_{\text {fluc }}(t) \tilde{\hat{L}}_{\text {fluc }}\left(t_{1}\right) \mid \tilde{\sigma}\left(t_{1}\right)\right) d t_{1} .
$$

An effective evolution equation for the spin dynamics including the collective effects of the fluctuations may be derived by averaging equation B3 over the ensemble

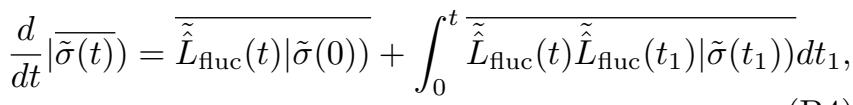

where the overline indicates the ensemble average. Although equation B4 does not appear to be much of a simplification, it builds the basis for subsequent approximation schemes. Following the excellent discussion by van Kampen ${ }^{3}$ we make use of the following key assumptions.

1) We may assume that fluctuating Liouvillian has a zero mean across the spin ensemble

$$
\overline{\hat{L}_{\text {fluc }}(t)}=-i \sum_{m} \overline{y_{m}(t)} \hat{Y}_{m}=0 .
$$

Any non-vanishing contributions may always be incorporated into the definition of $\hat{L}_{\text {coh }}$. The first term in equation B4 therefore vanishes

$$
\left.\frac{d}{d t} \mid \overline{\tilde{\sigma}(t)}\right)=\int_{0}^{t} \overline{\left.\hat{\hat{L}}_{\text {fluc }}(t) \tilde{\hat{L}}_{\text {fluc }}\left(t_{1}\right) \mid \tilde{\sigma}\left(t_{1}\right)\right)} d t_{1} .
$$

2) The ensemble average involving the fluctuating contributions and the density operator may be split into a product of averages

$$
\left.\left.\frac{d}{d t} \mid \overline{\tilde{\sigma}(t)}\right)=\int_{0}^{t} \overline{\tilde{\hat{L}}_{\text {fluc }}(t) \tilde{\hat{L}}_{\text {fluc }}\left(t_{1}\right)} \mid \overline{\tilde{\sigma}\left(t_{1}\right)}\right) d t_{1} .
$$

3) The density operator varies sufficiently slowly in time so that it is permissible to replace $\overline{\tilde{\sigma}\left(t_{1}\right)}$ by $\overline{\tilde{\sigma}(t)}$ in equation B7

$$
\left.\left.\frac{d}{d t} \mid \overline{\tilde{\sigma}(t)}\right)=\int_{0}^{t} \overline{\hat{\hat{L}}_{\text {fluc }}(t) \tilde{\hat{L}}_{\text {fluc }}\left(t_{1}\right)} \mid \overline{\tilde{\sigma}(t)}\right) d t_{1} .
$$


4) There exists a correlation time $\tau_{\mathrm{c}} \ll t$ short compared to the times we are interested in so that the limit of integration in equation B8 may be extended to infinity

$$
\left.\left.\frac{d}{d t} \mid \overline{\tilde{\sigma}(t)}\right)=\int_{0}^{+\infty} \overline{\hat{\hat{L}}_{\text {fluc }}(t) \tilde{\hat{L}}_{\text {fluc }}\left(t_{1}\right) \mid} \mid \overline{\tilde{\sigma}(t)}\right) d t_{1} .
$$

Expanding the fluctuating Liouvillian according to equation 8 leads to

$$
\begin{aligned}
& \left.\left.\frac{d}{d t} \mid \overline{\tilde{\sigma}(t)}\right)=\int_{0}^{+\infty} \overline{\hat{\hat{L}}_{\text {fluc }}(t) \tilde{\hat{L}}_{\text {fluc }}\left(t_{1}\right)} d t_{1} \mid \overline{\tilde{\sigma}(t)}\right) \\
& \left.=-\sum_{m n} \int_{0}^{+\infty} k_{m n}\left(t, t_{1}\right) \tilde{\hat{Y}}_{m}(t) \tilde{\hat{Y}}_{n}^{\dagger}\left(t_{1}\right) d t_{1} \mid \overline{\tilde{\sigma}(t)}\right)
\end{aligned}
$$

with $k_{m n}\left(t, t_{1}\right)$ denoting the correlation functions of the fluctuations

$$
k_{m n}\left(t, t_{1}\right)=\overline{y_{m}(t) y_{n}^{*}\left(t_{1}\right)} .
$$

For a stationary random process the correlation functions only depend upon the time difference $\tau=t-t_{1}$

$$
k_{m n}\left(t, t_{1}\right)=k_{m n}\left(t-t_{1}, 0\right):=k_{m n}(\tau) .
$$

If we define the ensemble averaged density operator as $\overline{\sigma(t)}=\rho(t)$ equation B10 may be expressed as follows within the original frame

$\left.\left.\frac{d}{d t} \mid \rho(t)\right)=\left\{\hat{L}_{\mathrm{coh}}-\sum_{m n} \int_{0}^{+\infty} k_{m n}(\tau) \tilde{\hat{Y}}_{m}(0) \tilde{\hat{Y}}_{n}^{\dagger}(\tau) d \tau\right\} \mid \rho(t)\right)$,

and is identical to equation 9 .

\section{Appendix C: Typical NMR correlation functions}

As discussed in section II A the relaxation superoperator $\hat{\Gamma}$ may be shown to depend upon the one-sided Fourier transformation of the correlation functions $k_{m n}(\tau)$

$$
J_{m n}(\omega)=\int_{0}^{+\infty} k_{m n}(\tau) \exp (+i \omega \tau) d \tau .
$$

In solution state NMR the most dominant contribution to nuclear spin relaxation originates from the stochastic modulation of nuclear spin interactions due to rotational Brownian motion. If the molecule undergoes isotropic rotational Brownian motion it may be shown that the correlation function between any two coupling parameters decays exponentially

$$
k_{m n}(\tau) \propto \exp \left(-|\tau| / \tau_{\mathrm{c}}\right),
$$

where $\tau_{\mathrm{c}}$ represents the rotational correlation time of the molecule. The spectral density of such an exponential correlation function is given by

$$
J_{m n}(\Omega) \propto \frac{\tau_{\mathrm{c}}}{1+\left(\Omega \tau_{\mathrm{c}}\right)^{2}}\left(1+i \Omega \tau_{\mathrm{c}}\right) .
$$

The real part of $J_{m n}(\Omega)$ is responsible for the dissipative character of $\hat{\Gamma}$. The imaginary part leads to a higherorder correction of the coherent Liouvillian, the so-called dynamic frequency shift ${ }^{4-7}$.

The rotational correlation time $\tau_{\mathrm{c}}$ for small molecules is typically on the order of nanoseconds to picoseconds. The transition frequencies $\Omega$ on the other hand are on the order several $\mathrm{MHz}$ for modern NMR spectrometers. As a result, a broad class of relevant NMR systems satisfies the so-called fast motion limit ${ }^{6,7}$

$$
\Omega \tau_{\mathrm{c}} \ll 1 \text {. }
$$

Within the fast-motion limit the spectral density of an exponential correlation function is well approximated by

$$
J_{m n}(\Omega) \simeq \frac{\tau_{\mathrm{c}}}{1+\left(\Omega \tau_{\mathrm{c}}\right)^{2}} \simeq \tau_{\mathrm{c}},
$$

so that dynamic frequency shifts may be safely neglected (at least to a first approximation).

\section{Appendix D: Long-lived operators at high-temperature and high-entropy}

For spin systems in contact with a thermal environment the quantum master equation ( equation 1 ) should be replaced by a "thermally corrected" master equation. Within the high-temperature and high-entropy approximation it is permissible to describe the evolution of the spin ensemble by the so-called inhomogeneous master equation ${ }^{4,68,109}$. This amounts to replacing $\mid \rho$ ) with its thermal deviation

$$
\left.\left.\left.\left.\left.\frac{d}{d t} \mid \rho\right)=\hat{L}(\mid \rho)-\mid \rho_{\text {eq }}\right)\right)=\left(\hat{L}_{\text {coh }}+\hat{\Gamma}\right)(\mid \rho)-\mid \rho_{\text {eq }}\right)\right),
$$

where $\rho_{\text {eq }}$ describes the equilibrium position of the density operator at some finite temperature $T$.

Following references $65-67$ the inhomogeneous character of equation D1 may be removed by defining a thermalisation superoperator $\hat{\Theta}$

$$
\left.\hat{\Theta}=\hat{\mathbb{1}}-\mid \rho_{\text {eq }}\right)(\mathbb{1} \mid .
$$

The projection operator $\left.\mid \rho_{\mathrm{eq}}\right)(\mathbb{1} \mid$ projects any nontraceless operator onto the thermal equilibrium state. Since the trace of any density operator is given by unity $(\operatorname{Tr}(\rho)=1)$, the dissipative part of equation D1 may be rearranged as follows

$$
\begin{aligned}
\left.\left.\hat{\Gamma}(\mid \rho)-\mid \rho_{\mathrm{eq}}\right)\right) & \left.=\hat{\Gamma}\left(\hat{\mathbb{1}}-\mid \rho_{\mathrm{eq}}\right)(\mathbb{\mathbb { 1 }} \mid) \mid \rho\right) \\
& \left.=\hat{\Gamma}^{\theta} \mid \rho\right),
\end{aligned}
$$

with the "thermalized" relaxation superoperator $\hat{\Gamma}^{\theta}$ given by

$$
\hat{\Gamma}^{\theta}=\hat{\Gamma}\left(\hat{\mathbb{1}}-\mid \rho_{\mathrm{eq}}\right)(\mathbb{1} \mid) .
$$

With these definitions in place it is straightforward to show that the following statement holds true. 
Proposition 4. Any long-lived spin operator $\Psi_{j}^{0} \in \operatorname{null}(\hat{\Gamma})$ is an element of $\Psi_{j}^{0} \in \operatorname{null}\left(\hat{\Gamma}^{\theta}\right)$ apart from the unity operator $\mathbb{1}$, which is being replaced by $\rho_{e q} \in \operatorname{null}\left(\hat{\Gamma}^{\theta}\right)$.

Proof. Consider any long-lived operator $\Psi_{j}^{0} \in \operatorname{null}(\hat{\Gamma})$ and consider its interaction with $\hat{\Gamma}^{\theta}$

$$
\begin{array}{rlr}
\left.\hat{\Gamma}^{\theta} \mid \Psi_{j}^{0}\right) & \left.=\hat{\Gamma}\left(\hat{\mathbb{1}}-\mid \rho_{\mathrm{eq}}\right)(\mathbb{1} \mid) \mid \Psi_{j}^{0}\right) \\
& \left.\left.=\hat{\Gamma} \mid \Psi_{j}^{0}\right)-\mid \rho_{\mathrm{eq}}\right)\left(\mathbb{1} \mid \Psi_{j}^{0}\right) & \\
& \left.=0-\mid \rho_{\mathrm{eq}}\right) \operatorname{Tr}\left(\Psi_{j}^{0}\right) & \\
& =0 & \text { since } \operatorname{Tr}\left(\Psi_{j}^{0}\right)=0 .
\end{array}
$$

We therefore have $\Psi_{j}^{0} \in \operatorname{null}\left(\hat{\Gamma}^{\theta}\right)$. Similarly consider the interaction of $\hat{\Gamma}^{\theta}$ with a normalised unity operator $N_{\mathbb{H}}^{-1} \mathbb{1}$

$$
\begin{aligned}
\left.N_{\mathbb{H}}^{-1} \hat{\Gamma}^{\theta} \mid \mathbb{1}\right) & \left.=N_{\mathbb{H}}^{-1} \hat{\Gamma}\left(\hat{\mathbb{1}}-\mid \rho_{\mathrm{eq}}\right)(\mathbb{1} \mid) \mid \Psi_{j}^{0}\right) \\
& \left.=N_{\mathbb{H}}^{-1} \mid \rho_{\mathrm{eq}}\right) \operatorname{Tr}(\mathbb{1}) \\
& \left.=\mid \rho_{\mathrm{eq}}\right)
\end{aligned}
$$$$
\text { since } \operatorname{Tr}(\mathbb{1})=N_{\mathbb{H}} \text {. }
$$

The unity operator is therefore not an element of $\operatorname{null}\left(\hat{\Gamma}^{\theta}\right)$, but the thermal equilibrium density operator is

$$
\begin{array}{rlr}
\left.\hat{\Gamma}^{\theta} \mid \rho_{\text {eq }}\right) & \left.=\hat{\Gamma}\left(\hat{\mathbb{1}}-\mid \rho_{\text {eq }}\right)(\mathbb{1} \mid) \mid \rho_{\text {eq }}\right) & \\
& \left.\left.=\hat{\Gamma}\left(\mid \rho_{\text {eq }}\right)-\mid \rho_{\text {eq }}\right) \operatorname{Tr}\left(\rho_{\text {eq }}\right)\right) & \\
& =0 & \text { since } \operatorname{Tr}\left(\rho_{\text {eq }}\right)=1 .
\end{array}
$$

As a result the thermalisation procedure given by equation D4 preserves the long-lived character of any operator $\Psi_{j}^{0}$.

\section{Appendix E: Schur Orthogonality Relations}

Proofs of the Schur orthogonality relations may be found in standard text books on group theory ${ }^{55-57}$, we simply summarise the result. Both the columns and rows of an irreducible matrix representation $D^{\Gamma}$ are orthogonal with respect to the group average

$$
\sum_{g \in G}^{|G|} D_{\gamma \mu}^{\Gamma_{j} *}(g) D_{\eta \nu}^{\Gamma_{k}}(g)=\frac{|G|}{d_{j}^{\Gamma}} \delta_{j k} \delta_{\gamma \eta} \delta_{\mu \nu},
$$

whereas the group average between different irreducible matrix representations $(j \neq k)$ vanishes.

\section{Appendix F: Spherical Tensor Operators}

An overview of dipolar spherical tensor operators for a spin pair $(i, \mathrm{j})$ is given in table I. Each spherical tensor operator is characterised by its superscripts $(i j)$ and its subscripts $(\mathrm{km})$. The superscripts indicate angular momentum coupling of spins $i$ and $j$, whereas the subscripts indicate its total angular momentum $k$ and $z$-angular momentum $m$.

\begin{tabular}{|c|c|}
\hline$m \backslash k$ & 2 \\
\hline \pm 2 & $\frac{1}{2} I_{i}^{ \pm} I_{j}^{ \pm}$ \\
\hline \pm 1 & $\mp \frac{1}{2}\left(I_{i}^{ \pm} I_{j z}+I_{i z} I_{j}^{ \pm}\right)$ \\
\hline 0 & $-\frac{1}{2 \sqrt{6}}\left(I_{i}^{+} I_{j}^{-}+I_{i}^{-} I_{j}^{+}-4 I_{i z} I_{j z}\right)$ \\
\hline
\end{tabular}

Table I. Dipolar spherical tensor operators for a coupled spin pairs $i$ and $j$.

\section{Appendix G: $\mathrm{S}_{3}$ Symmetry Adapted Basis}

Equation G1 summarises an $\mathrm{S}_{3}$ symmetry adapted basis of spin states for three identical spin- $1 / 2$ particles. Each basis state is characterised by a definite permutation symmetry under elements of the symmetric group $\mathrm{S}_{3}$. A convenient algorithm for the calculation of such bases has been outlined in reference 53 .

$$
\mathcal{B}_{\mathrm{S}_{3}}=\left\{\begin{aligned}
|1\rangle & =|\beta \beta \beta\rangle \\
|2\rangle & =\frac{1}{\sqrt{3}}(|\beta \beta \alpha\rangle+|\beta \alpha \beta\rangle+|\alpha \beta \beta\rangle) \\
|3\rangle & =\frac{1}{\sqrt{3}}(|\alpha \alpha \beta\rangle+|\alpha \beta \alpha\rangle+|\beta \alpha \alpha\rangle) \\
|4\rangle & =|\alpha \alpha \alpha\rangle \\
|5\rangle & =\frac{1}{\sqrt{3}}\left(|\beta \beta \alpha\rangle+\epsilon|\beta \alpha \beta\rangle+\epsilon^{*}|\alpha \beta \beta\rangle\right) \\
|6\rangle & =\frac{1}{\sqrt{3}}\left(|\beta \beta \alpha\rangle+\epsilon^{*}|\beta \alpha \beta\rangle+\epsilon|\alpha \beta \beta\rangle\right) \\
|7\rangle & =\frac{1}{\sqrt{3}}\left(|\alpha \alpha \beta\rangle+\epsilon|\alpha \beta \alpha\rangle+\epsilon^{*}|\beta \alpha \alpha\rangle\right) \\
|8\rangle & =\frac{1}{\sqrt{3}}\left(|\alpha \alpha \beta\rangle+\epsilon^{*}|\alpha \beta \alpha\rangle+\epsilon|\beta \alpha \alpha\rangle\right) \\
& \text { with } \quad \epsilon=\exp \left(-i \frac{2 \pi}{3}\right) .
\end{aligned}\right.
$$

\section{REFERENCES}

${ }^{1}$ J. Cavanagh, W. J. Fairbrother, A. G. Palmer III Professor, N. J. Skelton, and M. Rance, Protein NMR Spectroscopy: Principles and Practice, 2nd ed. (Academic Press, Amsterdam ; Boston, 2006).

${ }^{2}$ M. H. Levitt, Spin dynamics: basics of nuclear magnetic resonance (Wiley, 2001).

${ }^{3}$ N. G. Van Kampen, Stochastic Processes in Physics and Chemistry (Elsevier, 2007).

${ }^{4}$ A. Abragam, Principles of Nuclear Magnetism (Clarendon Press, Oxford, 1983).

${ }^{5}$ C. P. Slichter, Principles of Magnetic Resonance, 3rd ed., Springer Series in Solid-State Sciences (Springer-Verlag, Berlin Heidelberg, 1990).

${ }^{6}$ J. Kowalewski and L. Maler, Nuclear Spin Relaxation in Liquids: Theory, Experiments, and Applications, Second Edition, 2nd ed. (CRC Press, Boca Raton, 2017). 
${ }^{7}$ R. R. Ernst, G. Bodenhausen, and A. Wokaun, Principles of Nuclear Magnetic Resonance in One and Two Dimensions, Vol. 1 (Clarendon Press, 1990).

${ }^{8} \mathrm{~J}$. Jeener, in Advances in Magnetic and Optical Resonance, Vol. 10, edited by J. S. Waugh (Academic Press, 1982) pp. 1-51.

${ }^{9}$ M. Carravetta, O. G. Johannessen, and M. H. Levitt, Physical Review Letters 92, 153003 (2004).

${ }^{10} \mathrm{M}$. Carravetta and M. H. Levitt, Journal of the American Chemical Society 126, 6228 (2004).

${ }^{11} \mathrm{~K}$. Gopalakrishnan and G. Bodenhausen, Journal of Magnetic Resonance 182, 254 (2006).

${ }^{12}$ G. Pileio and M. H. Levitt, Journal of Magnetic Resonance 187, 141 (2007).

${ }^{13}$ P. Ahuja, R. Sarkar, P. R. Vasos, and G. Bodenhausen, The Journal of Chemical Physics 127, 134112 (2007), publisher: American Institute of Physics.

${ }^{14}$ S. Cavadini, J. Dittmer, S. Antonijevic, and G. Bodenhausen, Journal of the American Chemical Society 127, 15744 (2005).

${ }^{15}$ S. Cavadini and P. R. Vasos, Concepts in Magnetic Resonance Part A 32A, 68 (2008).

${ }^{16}$ R. Sarkar, P. Ahuja, P. R. Vasos, and G. Bodenhausen, ChemPhysChem 9, 2414 (2008).

${ }^{17}$ P. Ahuja, R. Sarkar, P. R. Vasos, and G. Bodenhausen, Journal of the American Chemical Society 131, 7498 (2009), publisher: American Chemical Society.

${ }^{18}$ W. S. Warren, E. Jenista, R. T. Branca, and X. Chen, Science 323, 1711 (2009).

${ }^{19}$ R. Sarkar, P. Ahuja, P. R. Vasos, and G. Bodenhausen, Physical Review Letters 104, 053001 (2010).

${ }^{20}$ M. C. D. Tayler and M. H. Levitt, Physical Chemistry Chemical Physics 13, 5556 (2011).

${ }^{21}$ M. C. D. Tayler, I. Marco-Rius, M. I. Kettunen, K. M. Brindle, M. H. Levitt, and G. Pileio, Journal of the American Chemical Society 134, 7668 (2012).

${ }^{22}$ N. Salvi, R. Buratto, A. Bornet, S. Ulzega, I. Rentero Rebollo, A. Angelini, C. Heinis, and G. Bodenhausen, Journal of the American Chemical Society 134, 11076 (2012).

${ }^{23}$ Y. Feng, R. M. Davis, and W. S. Warren, Nature Physics 8, 831 (2012), number: 11 Publisher: Nature Publishing Group.

${ }^{24}$ M. H. Levitt, Annual Review of Physical Chemistry 63, 89 (2012).

${ }^{25}$ G. Pileio, S. Bowen, C. Laustsen, M. C. D. Tayler, J. T. HillCousins, L. J. Brown, R. C. D. Brown, J. H. Ardenkjaer-Larsen, and M. H. Levitt, Journal of the American Chemical Society 135, 5084 (2013), publisher: American Chemical Society.

${ }^{26}$ I. Marco-Rius, M. C. D. Tayler, M. I. Kettunen, T. J. Larkin, K. N. Timm, E. M. Serrao, T. B. Rodrigues, G. Pileio, J. H. Ardenkjaer-Larsen, M. H. Levitt, and K. M. Brindle, NMR in Biomedicine 26, 1696 (2013).

${ }^{27}$ A. Bornet, X. Ji, D. Mammoli, B. Vuichoud, J. Milani, G. Bodenhausen, and S. Jannin, Chemistry - A European Journal 20, 17113 (2014), publisher: John Wiley \& Sons, Ltd.

${ }^{28}$ B. Vuichoud, J. Milani, A. Bornet, R. Melzi, S. Jannin, and G. Bodenhausen, The Journal of Physical Chemistry B 118, 1411 (2014), publisher: American Chemical Society.

${ }^{29}$ G. Stevanato, J. T. Hill-Cousins, P. Håkansson, S. S. Roy, L. J. Brown, R. C. D. Brown, G. Pileio, and M. H. Levitt, Angewandte Chemie International Edition 54, 3740 (2015).

${ }^{30}$ M. H. Levitt, Journal of Magnetic Resonance 262, 91 (2016).

${ }^{31} \mathrm{M}$. Icker and S. Berger, Journal of Magnetic Resonance 219, 1 (2012).

${ }^{32}$ B. Meier, J.-N. Dumez, G. Stevanato, J. T. Hill-Cousins, S. S. Roy, P. Håkansson, S. Mamone, R. C. D. Brown, G. Pileio, and M. H. Levitt, Journal of the American Chemical Society 135, 18746 (2013).

${ }^{33}$ S. S. Roy, J.-N. Dumez, G. Stevanato, B. Meier, J. T. HillCousins, R. C. D. Brown, G. Pileio, and M. H. Levitt, Journal of Magnetic Resonance 250, 25 (2015).

${ }^{34}$ J.-N. Dumez, B. Vuichoud, D. Mammoli, A. Bornet, A. C. Pinon, G. Stevanato, B. Meier, G. Bodenhausen, S. Jannin, and M. H. Levitt, The Journal of Physical Chemistry Letters 8 , 3549 (2017).

${ }^{35}$ B. Meier, K. Kouřil, C. Bengs, H. Kouřilová, T. C. Barker, S. J. Elliott, S. Alom, R. J. Whitby, and M. H. Levitt, Physical Review Letters 120, 266001 (2018).

${ }^{36}$ A. Jhajharia, E. M. M. Weber, J. G. Kempf, D. Abergel, G. Bodenhausen, and D. Kurzbach, The Journal of Chemical Physics 146, 041101 (2017).

${ }^{37}$ T. Kress, A. Walrant, G. Bodenhausen, and D. Kurzbach, The Journal of Physical Chemistry Letters 10, 1523 (2019).

${ }^{38}$ S. S. Roy, K. M. Appleby, E. J. Fear, and S. B. Duckett, The Journal of Physical Chemistry Letters 9, 1112 (2018), publisher: American Chemical Society.

${ }^{39}$ S. J. Elliott, B. Meier, B. Vuichoud, G. Stevanato, L. J. Brown, J. Alonso-Valdesueiro, L. Emsley, S. Jannin, and M. H. Levitt, Physical Chemistry Chemical Physics 20, 9755 (2018).

${ }^{40}$ J. Eills, W. Hale, M. Sharma, M. Rossetto, M. H. Levitt, and M. Utz, Journal of the American Chemical Society 141, 9955 (2019), publisher: American Chemical Society.

${ }^{41}$ B. Procacci, S. S. Roy, P. Norcott, N. Turner, and S. B. Duckett, Journal of the American Chemical Society 140, 16855 (2018), publisher: American Chemical Society.

${ }^{42}$ W. Iali, S. S. Roy, B. J. Tickner, F. Ahwal, A. J. Kennerley, and S. B. Duckett, Angewandte Chemie 131, 10377 (2019).

${ }^{43}$ M. Carravetta and M. H. Levitt, The Journal of Chemical Physics 122, 214505 (2005).

${ }^{44}$ E. Vinogradov and A. K. Grant, Journal of Magnetic Resonance 188, 176 (2007).

${ }^{45}$ A. K. Grant and E. Vinogradov, Journal of Magnetic Resonance 193, 177 (2008).

${ }^{46}$ G. Pileio and M. H. Levitt, The Journal of Chemical Physics 130, 214501 (2009).

${ }^{47}$ P. Ahuja, R. Sarkar, P. R. Vasos, and G. Bodenhausen, Chem. Phys. Chem. 10, 2217 (2009).

${ }^{48}$ H. J. Hogben, P. J. Hore, and I. Kuprov, Journal of Magnetic Resonance 211, 217 (2011).

${ }^{49}$ Y. Feng, T. Theis, T.-L. Wu, K. Claytor, and W. S. Warren, The Journal of Chemical Physics 141, 134307 (2014), publisher: American Institute of Physics.

${ }^{50}$ G. Stevanato, S. S. Roy, J. Hill-Cousins, I. Kuprov, L. J. Brown, R. C. D. Brown, G. Pileio, and M. H. Levitt, Physical Chemistry Chemical Physics 17, 5913 (2015), publisher: The Royal Society of Chemistry.

${ }^{51} \mathrm{G}$. Stevanato, Long-lived states in multi-spin systems, phd, University of Southampton (2015).

52 J.-N. Dumez, P. Håkansson, S. Mamone, B. Meier, G. Stevanato, J. T. Hill-Cousins, S. S. Roy, R. C. D. Brown, G. Pileio, and M. H. Levitt, The Journal of Chemical Physics 142, 044506 (2015).

${ }^{53}$ C. Bengs, The Journal of Chemical Physics 152, 054106 (2020), publisher: American Institute of Physics.

${ }^{54}$ K. L. Ivanov, T. Kress, M. Baudin, D. Guarin, D. Abergel, G. Bodenhausen, and D. Kurzbach, The Journal of Chemical Physics 149, 054202 (2018).

${ }^{55}$ M. Hamermesh, Group Theory and Its Application to Physical Problems, reprinted ed edition ed. (Dover Publications Inc., New York, 1990).

${ }^{56}$ J. F. Cornwell, Group Theory in Physics, Volume 1: An Introduction, abridged edition ed. (Academic Press, San Diego, 1997).

${ }^{57}$ J.-Q. Chen, J. Ping, and F. Wang, Group Representation Theory for Physicists, 2nd ed. (World Scientific, 2002).

${ }^{58}$ A. Barenco, A. Berthiaume, D. Deutsch, A. Ekert, R. Jozsa, and C. Macchiavello, SIAM Journal on Computing 26, 1541 (1997), publisher: Society for Industrial and Applied Mathematics.

${ }^{59}$ D. A. Lidar, I. L. Chuang, and K. B. Whaley, Physical Review Letters 81, 2594 (1998), publisher: American Physical Society.

${ }^{60} \mathrm{X}$. Wang, M. Byrd, and K. Jacobs, Physical Review A 87, 012338 (2013), publisher: American Physical Society. 
${ }^{61} \mathrm{X}$. Wang, M. Byrd, and K. Jacobs, Physical Review Letters 116, 090404 (2016), publisher: American Physical Society.

${ }^{62}$ P. S. Hubbard, Physical Review 131, 1155 (1963), publisher: American Physical Society.

${ }^{63}$ D. E. Woessner, The Journal of Chemical Physics 37, 647 (1962), publisher: American Institute of Physics.

${ }^{64}$ P. S. Hubbard, Physical Review A 6, 2421 (1972).

${ }^{65}$ M. H. Levitt and L. Di Bari, Physical Review Letters 69, 3124 (1992).

${ }^{66}$ M. H. Levitt and L. Di Bari, Bulletin of Magnetic Resonance 16, 94 (1994).

${ }^{67}$ T. O. Levante and R. R. Ernst, Chemical Physics Letters 241 73 (1995)

${ }^{68}$ C. Bengs and M. H. Levitt, Journal of Magnetic Resonance , 106645 (2019)

${ }^{69}$ G. Lindblad, Communications in Mathematical Physics 48, 119 (1976).

70 A. Kossakowski, Reports on Mathematical Physics 3, 247 (1972).

${ }^{71}$ V. Gorini, A. Kossakowski, and E. C. G. Sudarshan, Journal of Mathematical Physics 17, 821 (1976).

${ }^{72}$ B. C. Hall, Lie Groups, Lie Algebras, and Representations: An Elementary Introduction, 2nd ed., Graduate Texts in Mathematics (Springer International Publishing, 2015).

${ }^{73} \mathrm{~K}$. Tapp, Matrix Groups for Undergraduates, 2nd ed. (American Mathematical Society, Providence, Rhode Island, 2016).

${ }^{74}$ H.-P. Breuer and F. Petruccione, The Theory of Open Quantum Systems (Oxford University Press, 2007).

${ }^{75}$ G. Schaller, Open Quantum Systems Far from Equilibrium, Lecture Notes in Physics (Springer International Publishing, 2014).

${ }^{76}$ B. E. Sagan, The Symmetric Group: Representations, Combinatorial Algorithms, and Symmetric Functions, 2nd ed. (Springer, New York, 2001).

${ }^{77}$ M. H. Levitt, Journal of Magnetic Resonance 306, 69 (2019).

${ }^{78}$ G. Pileio, ed., Long-lived Nuclear Spin Order: Theory and Applications, 1st ed. (Royal Society of Chemistry, S.l., 2020).

${ }^{79}$ B. Meier, Magnetic Resonance in Chemistry 56, 610 (2018).

${ }^{80}$ C. Dietrich, J. Wissel, J. Knoche, O. Lorenz, and J. Matysik, Molecular Physics 117, 2772 (2019).

${ }^{81}$ R. W. Haase and P. H. Butler, Journal of Physics A: Mathematical and General 17,61 (1984).

${ }^{82}$ D. J. Rowe, M. J. Carvalho, and J. Repka, Reviews of Modern Physics 84, 711 (2012).

${ }^{83}$ M. C. D. Tayler, S. Marie, A. Ganesan, and M. H. Levitt, Journal of the American Chemical Society 132, 8225 (2010), publisher: American Chemical Society.

${ }^{84}$ M. C. D. Tayler and M. H. Levitt, Physical Chemistry Chemical Physics 13, 9128 (2011), publisher: Royal Society of Chemistry.

${ }^{85}$ S. J. Elliott, C. Bengs, L. J. Brown, J. T. Hill-Cousins, D. J. O'Leary, G. Pileio, and M. H. Levitt, The Journal of Chemical Physics 150, 064315 (2019).

${ }^{86}$ A. S. Kiryutin, H. Zimmermann, A. V. Yurkovskaya, H.-M. Vieth, and K. L. Ivanov, Journal of Magnetic Resonance 261, 64 (2015).

${ }^{87}$ G. Pileio, J.-N. Dumez, I.-A. Pop, J. T. Hill-Cousins, and R. C. D. Brown, Journal of Magnetic Resonance 252, 130 (2015).

${ }^{88}$ G. Pileio and S. Ostrowska, Journal of Magnetic Resonance (San Diego, Calif.: 1997) 285, 1 (2017).
${ }^{89}$ M. C. Tourell, I.-A. Pop, L. J. Brown, R. C. D. Brown, and G. Pileio, Physical Chemistry Chemical Physics 20, 13705 (2018).

${ }^{90}$ J. Eills, E. Cavallari, R. Kircher, G. D. Matteo, C. Carrera, L. Dagys, M. H. Levitt, K. L. Ivanov, S. Aime, F. Reineri, K. Münnemann, D. Budker, G. Buntkowsky, and S. Knecht, Angewandte Chemie International Edition 60, 6791 (2021).

${ }^{91}$ M. Emondts, M. Ledbetter, S. Pustelny, T. Theis, B. Patton, J. Blanchard, M. Butler, D. Budker, and A. Pines, Physical Review Letters 112, 077601 (2014), publisher: American Physical Society.

${ }^{92}$ K. V. Kovtunov, M. L. Truong, D. A. Barskiy, I. V. Koptyug, A. M. Coffey, K. W. Waddell, and E. Y. Chekmenev, Chemistry - A European Journal 20, 14629 (2014).

${ }^{93}$ I. V. Zhukov, A. S. Kiryutin, A. V. Yurkovskaya, Y. A. Grishin, H.-M. Vieth, and K. L. Ivanov, Physical Chemistry Chemical Physics 20, 12396 (2018), publisher: Royal Society of Chemistry.

${ }^{94}$ A. S. Kiryutin, M. S. Panov, A. V. Yurkovskaya, K. L. Ivanov, and G. Bodenhausen, ChemPhysChem 20, 766 (2019).

${ }^{95}$ P. R. Vasos, A. Comment, R. Sarkar, P. Ahuja, S. Jannin, J.-P. Ansermet, J. A. Konter, P. Hautle, B. v. d. Brandt, and G. Bodenhausen, Proceedings of the National Academy of Sciences 106, 18469 (2009).

${ }^{96}$ S. S. Roy, P. J. Rayner, P. Norcott, G. G. R. Green, and S. B. Duckett, Physical Chemistry Chemical Physics 18, 24905 (2016).

${ }^{97}$ T. Theis, G. X. Ortiz, A. W. J. Logan, K. E. Claytor, Y. Feng, W. P. Huhn, V. Blum, S. J. Malcolmson, E. Y. Chekmenev, Q. Wang, and W. S. Warren, Science Advances 2, e1501438 (2016).

${ }^{98}$ Z. Zhou, J. Yu, J. F. P. Colell, R. Laasner, A. Logan, D. A. Barskiy, R. V. Shchepin, E. Y. Chekmenev, V. Blum, W. S. Warren, and T. Theis, The Journal of Physical Chemistry Letters 8, 3008 (2017), publisher: American Chemical Society.

${ }^{99}$ K. F. Sheberstov, H.-M. Vieth, H. Zimmermann, B. A. Rodin, K. L. Ivanov, A. S. Kiryutin, and A. V. Yurkovskaya, Scientific Reports 9, 20161 (2019), number: 1 Publisher: Nature Publishing Group.

${ }^{100}$ K. Miyanishi, N. Ichijo, M. Motoyama, A. Kagawa, M. Negoro, and M. Kitagawa, Quantum Science and Technology 5, 025004 (2020), publisher: IOP Publishing.

${ }^{101}$ G. A. Álvarez, A. Ajoy, X. Peng, and D. Suter, Physical Review A 82, 042306 (2010), publisher: American Physical Society.

${ }^{102}$ G. S. Uhrig, J. Hackmann, D. Stanek, J. Stolze, and F. B. Anders, Physical Review B 90, 060301 (2014), publisher: American Physical Society.

${ }^{103}$ U. Seifert, P. Bleicker, P. Schering, A. Faribault, and G. S. Uhrig, Physical Review B 94, 094308 (2016), publisher: American Physical Society.

${ }^{104}$ N. Wu, N. Fröhling, X. Xing, J. Hackmann, A. Nanduri, F. B. Anders, and H. Rabitz, Physical Review B 93, 035430 (2016), publisher: American Physical Society.

${ }^{105}$ P. Mazur, Physica 43, 533 (1969).

${ }^{106}$ M. Suzuki, Physica 51, 277 (1971).

${ }^{107}$ W. R. Inc., "Mathematica, Version 12.2," (2020).

${ }^{108}$ C. Bengs and M. H. Levitt, Magnetic Resonance in Chemistry 56, 374 (2018).

${ }^{109} \mathrm{M}$. H. Levitt and C. Bengs, Magnetic Resonance Discussions, 1 (2021), publisher: Copernicus GmbH. 